\title{
TabReformer: Unsupervised Representation Learning for Erroneous Data Detection
}

\author{
MONA NASHAAT, AINDRILA GHOSH, and JAMES MILLER, Electrical and Computer \\ Engineering, University of Alberta, Edmonton, Alberta, Canada \\ SHAIKH QUADER, IBM Canada Software Lab, IBM Canada, Toronto, Ontario, Canada
}

\begin{abstract}
Error detection is a crucial preliminary phase in any data analytics pipeline. Existing error detection techniques typically target specific types of errors. Moreover, most of these detection models either require userdefined rules or ample hand-labeled training examples. Therefore, in this article, we present TabReformer, a model that learns bidirectional encoder representations for tabular data. The proposed model consists of two main phases. In the first phase, TabReformer follows encoder architecture with multiple self-attention layers to model the dependencies between cells and capture tuple-level representations. Also, the model utilizes a Gaussian Error Linear Unit activation function with the Masked Data Model objective to achieve deeper probabilistic understanding. In the second phase, the model parameters are fine-tuned for the task of erroneous data detection. The model applies a data augmentation module to generate more erroneous examples to represent the minority class. The experimental evaluation considers a wide range of databases with different types of errors and distributions. The empirical results show that our solution can enhance the recall values by $32.95 \%$ on average compared with state-of-the-art techniques while reducing the manual effort by up to $48.86 \%$.
\end{abstract}

CCS Concepts: • Information systems $\rightarrow$ Data cleaning; • Computing methodologies $\rightarrow$ Neural networks; Anomaly detection;

Additional Key Words and Phrases: Error detection, transformers, bidirectional encoder, data augmentation

ACM Reference format:

Mona Nashaat, Aindrila Ghosh, James Miller, and Shaikh Quader. 2020. TabReformer: Unsupervised Representation Learning for Erroneous Data Detection. ACM/IMS Trans. Data Sci. 2, 3, Article 18 (May 2021), 29 pages.

https://doi.org/10.1145/3447541

\section{INTRODUCTION}

Data and analytics have become one of the top growth opportunities for business. Data-driven decision making has proven to lead to better accountability for every organization. However, poor

\footnotetext{
Authors' addresses: M. Nashaat (corresponding author), A. Ghosh, and J. Miller, Electrical and Computer Engineering, University of Alberta, 116 St \& 85 Ave, Edmonton, AB T6G 2R3, Canada; emails: \{nashaata, aindrila, jimm\}@ualberta.ca; S. Quader, IBM Canada, Software Lab, IBM Canada, 8200 Warden Ave, Unionville, ON L6G 1C7, Canada; email: shaikhq@ca.ibm.com.

Permission to make digital or hard copies of all or part of this work for personal or classroom use is granted without fee provided that copies are not made or distributed for profit or commercial advantage and that copies bear this notice and the full citation on the first page. Copyrights for components of this work owned by others than the author(s) must be honored. Abstracting with credit is permitted. To copy otherwise, or republish, to post on servers or to redistribute to lists, requires prior specific permission and/or a fee. Request permissions from permissions@acm.org.

(c) 2020 Copyright held by the owner/author(s). Publication rights licensed to ACM.

2577-3224/2020/05-ART18 \$15.00

https://doi.org/10.1145/3447541
}

ACM/IMS Transactions on Data Science, Vol. 2, No. 3, Article 18. Publication date: May 2021. 
data quality can have adverse impacts on businesses and cause significant financial loss [1]. Thus, data cleansing has become an essential prerequisite for developing business intelligence solutions. Data cleansing refers to the process of identifying and rectifying inaccurate records in databases. The procedure involves two practices intending to produce high-quality data. First, erroneous data cells are identified; then, data correction routines [2] are applied to fix or remove the corrupted data. Typically, errors originate from diverse sources, such as syntax errors and type conversions. Therefore, error detection can be challenging since it requires running multiple detection algorithms to cover various error types. Moreover, dealing with big data [1] makes manual error detection prohibitively expensive. Data quality issues are considered to be the main enemy for machine learning and analytics. Since "garbage-in, garbage-out" forms an ongoing threat for machine learning models, inaccurate data has severe consequences for businesses [3]. Therefore, error detection is considered to be a critical step to maintain a stable analytics pipeline.

As a result, there have been numerous studies to investigate automating the process of detecting erroneous data. A lot of research is targeted to handle outlier detection [4-7], rule violations [8, 9], and duplicate data detection [10]. Rule-based systems [8,9] count on the identification of a set of data quality rules using integrity constraints [11] to specify functional dependencies or other constraints that may define data quality in the given domain. Although these techniques are proven to be effective in many situations [12], they cannot be considered as conclusive for many reasons. First, each of these methods is customized only to detect specific types of erroneous data. Hence, their performance is not guaranteed in many situations in which diverse forms of errors coexist in the same database [13]. Second, some of these approaches are effective only with specific configurations of the examined data. For example, most outlier detection methods are susceptible to imbalanced distributions or high-dimensional datasets [7]. With large datasets in a high-dimensional space, classifiers cannot separate outliers from the original data using the limited number of outliers available during training. Third, as previous research [13] points out, most of these systems are evaluated using only synthetic data, which might not be enough to test their suitability in real-world situations.

Finally, all of these solutions still require some input from the end-user. For example, rule-based systems $[8,9]$ oblige the user to write integrity constraints [9], such as denial constraints [2]. These systems then utilize these rules to detect violating cells that do not comply with these specified rules. However, writing integrity constraints requires an adequate level of domain knowledge alongside the technical expertise needed to write regular expressions [9]. Also, outlier detection methods require precise identification of outlier thresholds. Existing thresholding techniques rely on statistics, making them considerably biased when dealing with data with many outliers [14]. Hence, end-users' input may be needed to evaluate the choice of these thresholds, which can be time-consuming.

Alternatively, while trying to address some of these challenges, some research $[3,15,16]$ has recently investigated the effectiveness of applying machine learning to the problem of error detection. Since detecting erroneous cells can be seen as a binary classification problem, a learning model can be trained to differentiate faulty values from correct ones. Furthermore, the expressive power of sophisticated models such as neural networks can overcome error heterogeneity and detect multiple classes of errors. Additionally, except for training data, learning models do not require additional inputs from the user. However, several challenges regarding applying machine learning to error detection remain to be addressed. For instance, previous techniques $[16,17] \mathrm{em}-$ ploy supervised learning, requiring a considerable amount of labeled data to train such models. Alternatively, even though some techniques $[17,18]$ apply sampling strategies to reduce the volume of labeled examples, the burden required for feature engineering is believed to be substantial [16]. 


\begin{tabular}{|c|c|c|c|c|}
\cline { 2 - 5 } \multicolumn{1}{c|}{} & ID & Airport Name & City & State \\
\hline$l_{1}$ & $1055 \mid$ & Bethel Airport & Beathel & AK \\
\hline$l_{2}$ & 14709 & Deadharse Airport & Deadhorse & AM \\
\hline$l_{3}$ & 12992 & Adams Field & Little Rack & AR \\
\hline$l_{4}$ & 10800 & Bab Hope & Burbank & AR \\
\hline
\end{tabular}

Fig. 1. An example dataset with errors.

A closer look at the sources of errors, however, states that attention [19] matters. Attention mechanisms [19] are a recent technique targeted at representation learning. Attention-based networks consider the dependency relationships between different parts of the input vector. Thus, they learn interdependent representations, which are essential to solving many tasks, such as speech recognition [20] and document summarization [21]. Comparably, when considering tabular data, attentive models can observe different levels of dependencies between the input features, which can be effectively employed to detect erroneous data.

As an example, Figure 1 shows a snippet of the Airports table from the Flights database [22]. Examples for misspelled values are shown in the figure (i.e., the city name in $l_{1}$ and the state name in $l_{2}$ should be spelled as "Bethel" and "AR," respectively). Also, a value swapping error appears in $l_{4}$ : i.e., Burbank is in California state (CA), not in Arkansas (AR). Most of these errors go beyond traditional rule-based systems since the errors cannot be detected using traditional integrity constraints. One expensive solution to catch such errors is to provide the dataset with many constant conditional functional dependencies, such as [Airport_Name ([airport name $=$ "Bethel Airport"] $\rightarrow$ [city = "Bethel"]) and as [City ([city = "Burbank"] $\rightarrow$ [state = "AR"]). Alternatively, since these errors are related to the data context, we believe that an attentive-based network can employ data representation to reflect on interattribute dependencies and find these errors.

Hence, inspired by the significant improvements that attention techniques have achieved in language understanding tasks [23], we introduce TabReformer, a model that applies unsupervised representation learning to model attribute dependencies in tabular data. Unsupervised representation learning generally aims at discovering data representations that are beneficial for consequent tasks. In TabReformer, discovering data representation is accomplished in the first phase without the need to supervised labels. On the other hand, TabReformer is considered the first attempt to apply transformers to structured data with the aim of error detection. A component overview of the proposed framework is illustrated in Figure 2. As the figure shows, the model has two main phases. The first phase trains a bidirectional encoder representation model with a Masked Data Model (MDM) objective. In this phase, we randomly replace a percentage of the input features with a special masked token. Then, the model is trained to classify the masked cells. In the second phase, we fine-tune the system parameters with the task of erroneous data detection. To minimize the manual effort in providing training data, the system applies data augmentation that takes a set of correct data points and returns erroneous synthetic examples. The code of the framework, along with the trained models created during the evaluation, are publicly available at https://github.com/MonaNashaat/TabReformer, which allows the reader to replicate all of the results produced in this article.

To evaluate the proposed model, we compare its performance with five state-of-the-art techniques for error detection and data repairing $[3,9,16,17]$. The primary contributions of this research can be summarized as follows: 


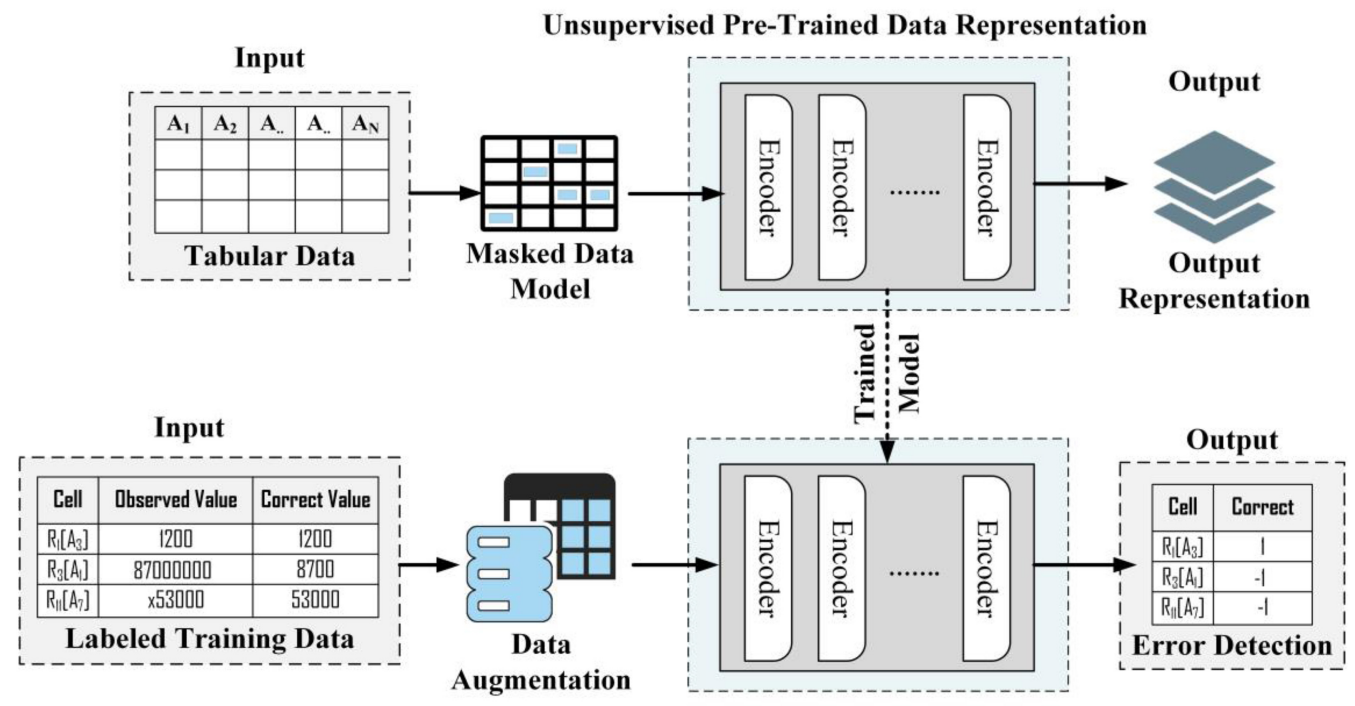

Fine-tuning using Augmented Labeled Data

Fig. 2. A component overview of TabReformer.

- An end-to-end framework is introduced for representation learning for structured data. The system applies bidirectional encoder representations to model the data and detects erroneous values. The proposed method's architecture includes a novel learning objective for tabular data and a data augmentation module. The data augmentation phase does not require any user-defined parameters; that is, it is fully automated and assumes no domainspecific knowledge. Instead, the transformation functions and the augmentation strategy are concluded from the input data. Regarding the training and the fine-tuning phases, the system requires a set of hyperparameters. In TabReformer, these parameters are set using cross-validation and been validated using a set of real-world datasets.

- We apply an extensive set of experiments to evaluate the proposed system against stateof-the-art techniques. The evaluation uses six datasets of varying size, dimensionality, and error rates and distributions. The experiments also involve a micro-benchmark to evaluate the impact of different design decisions implemented in the proposed method.

The article is structured as follows. In Section 2, we present an overview of the background related to this research. We then describe the individual components of TabReformer in Section 3. Section 4 defines the evaluation setup and experimental results. Section 5 reviews related work and Section 6 presents our conclusions.

\section{BACKGROUND}

In this section, we review methods for error detection in database systems and discuss data augmentation for resolving data imbalance. We then present transformers as a new architecture of attentive-based neural networks that have been gaining popularity in many applications, such as machine translation and language modeling.

\subsection{Error Detection}

There has been extensive research into error detection and data cleaning algorithms to identify and repair possible data errors. According to the error sources, we categorize existing error 
detection methods into (1) qualitative methods and (2) quantitative methods. Qualitative methods are rule-based approaches that rely on a set of data quality rules and use them to specify violating cells. Denial constraints [9] can be used to determine data quality rules in the form of first-order formulae that incorporate different types of integrity constraints. These constraints can be either supplied by domain experts [8, 28], or (potentially) automatically derived from the data [29].

Existing tools $[8,9]$ focus on analyzing these constraints and defining data inconsistencies with these rules. For example, Schelter et al. [8] propose a declarative API that allows a user to define database constraints. Then, the approach executes an algorithm for constraint validation to detect violating data. Similarly, authors in [9] proposed NADEEF as a prototype that follows a similar pipeline of collecting user-specified constraints. These constraints are compiled to detect erroneous data and select the most appropriate data repair algorithm.

Alternatively, pattern mining approaches attempt to discover the syntactic and semantic characterizations of the data. One technique for pattern discovery is inducing functional dependencies from the data [30-32]. Functional dependencies are considered a special form of denial constraints [30] and are commonly used to specify business rules. For example, tuples with the same value for longitude must share the same time zone. Existing research [31] has studied repeated patterns in the data and formalize them into functional dependencies to suggest better repair solutions. Another study [32] focuses on deriving such dependencies with the presence of erroneous data; the method [32] introduces a new class of integrity constraints that can infer dependencies between data attributes even if a portion of the attributes violates these dependencies.

Quantitative methods, on the other hand, employ statistical techniques to identify unusual behavior in the data. One good example of such techniques is outlier detection. Existing research [4-7] applies data modeling approaches to detect outliers in numerical data, e.g., Gaussian mixture models [4] or histogram modeling [5]. Moreover, recent research has applied machine learning techniques, such as unsupervised learning [6] and active learning [7], to detect outliers in relational databases. For example, Riahi and Schulte [6] propose a technique to learn a model for outlier detection using Bayesian networks. The method integrates exception mining with statistical-relational learning to detect outliers in relational data.

However, there are vital questions that are still not addressed in these approaches. For example, all of these techniques require end-user intervention in various time-consuming and nontrivial stages along the way. For example, rule-based systems require users to provide inputs such as algorithm configuration, data quality constraints, and the verification of final results [13]. Although some efforts $[11,33]$ try to automatically derive denial constraints, these approaches still depend on the user to provide an appropriate error threshold. Moreover, these tools can be computationally costly due to the enormous search space of the constraints [11]. Also, since each of these techniques is designed to deal with specific types of errors, real-world applications may require using a combination of these detection methods. However, integrating the outputs of these tools requires significant engineering, which often becomes the user's responsibility. Finally, the performance of these combinations depends on the weights assigned to every result for each technique. Thus, the robustness of these methods to capture errors in real-world databases is still to be verified, which provides the motivation of this research.

\subsection{Data Augmentation}

Data augmentation is an approach that allows practitioners to economically generate data to enhance the input variety (and volume) presented to machine learning models. Typically, neural networks require a massive amount of labeled data to model the underlying distribution of the general population. Training deep learning models with a small training dataset can result in overfitting. In such a scenario, the model memorizes the input examples and their corresponding outputs. 
Therefore, adding more data (different) examples offers a broader description of the general population from which the model can be learned. Hence, data augmentation presents a reasonable solution for obtaining more training examples when acquiring real labeled data, which can be time-consuming or prohibitively expensive.

Data augmentation assumes that more information can be obtained by applying a set of transformations to the original dataset. Typically, data augmentation consists of two elements: (1) a set of transformation functions that, when applied to the original data, can generate additional examples; and (2) a data augmentation strategy that determines how these functions should be applied to the data. Many approaches $[34,35]$ are proposed to specify augmentation policies for different classification tasks. For example, Cubuk et al. [35] present a search algorithm to automatically find data augmentation strategies. The algorithm applies reinforcement learning [36] to find the optimal policy among predefined geometric transformations sets. However, most of these techniques are focused on specific applications, such as image analysis [35] or speech recognition [37].

Several questions are raised regarding the cost of these approaches [34, 35]. For instance, previous studies [38,39] stated that the algorithm proposed in [35] requires training a massive number of models, which can take thousands of GPU hours. As a result, this research aims to investigate the usefulness of data augmentation approaches for structured tabular data to reduce manual efforts in the context of erroneous data detection.

\subsection{Transformers}

Transformers are a novel (neural network) architecture that was first presented in [19]. The architecture applies an attention mechanism [40] to enable transformers to understand complex structures such as natural language. The attention mechanism was initially proposed for machine translation tasks so that it can process an input sentence and decide, for each input token, which other parts of the input are essential. The mechanism extracts keywords that are important to sentence semantics. Thus, the network can execute translation more effectively. Self-attention [23] is an attention mechanism that aims to derive a representation of an input sequence by estimating relationships between items in this sequence. This mechanism has shown significant advances in natural language processing, such as abstractive summarization [21] and language modeling [23].

Similarly, transformers follow the same structure of sequence-to-sequence models [41] by utilizing an encoder-decoder architecture. The encoder processes the input and maps it to a single latent vector denoting the whole input sequence. The input first goes through a self-attention layer to allow the encoder to look at each word (token) in the input sequence. Then, the output of the self-attention layer is passed to feed-forward (neural) networks, which process each of these encodings individually [19]. The encoder's output is then fed to a decoder, which unpacks the encoding into a target sequence (e.g., the same sentence translated in a different language). The decoder has a similar structure to the encoder; however, it has an additional attention layer that enables the decoder to focus on relevant parts of the input sentence.

Until now, transformers have shown improvements in many tasks, including machine translation [42] and language understanding [23]. One example of such a transformer is BERT [23]. BERT applies an encoder representation using transformers to execute bidirectional training for language modeling. Motivated by BERT, recent research [42-44] has examined different configurations for transformer networks to enhance their capabilities. For example, Dai et al. [42] propose an enhancement that allows transformers to learn a language model beyond fixed-length contexts. Other research teams $[43,44]$ have proposed some design changes to enhance BERT's performance. While some of these changes [44] aim to reduce the number of parameters to enhance memory consumption, others [43] modified BERT's hyperparameters to enhance the overall performance. All of these models [23, 42-44] have focused on language modeling-to the best of 
our knowledge-no previous research has investigated the effectiveness of attention-based models for error detection in tabular data. Hence, TabReformer can be considered a novel research hypothesis that transformer-based approaches can be successfully applied to this new unexplored problem area.

\section{TABREFORMER: THE PROPOSED FRAMEWORK}

In the following subsections, we describe the architecture of TabReformer. Section 3.1 formulates the problem statement for error detection in databases. Section 3.2 describes, in detail, the phases of the proposed solution.

\subsection{Problem Statement}

TabReformer aims at classifying erroneous values in a database. Relational databases formally consist of a set of tables, while each table D comprises: a set of attributes $A=\left\{\mathrm{a}_{\mathrm{i}}\right\}_{\mathrm{i}=1}^{\mathrm{N}}$ (columns), and a group of tuples $\mathrm{L}=\left\{\mathrm{l}_{\mathrm{i}}\right\}_{\mathrm{i}=1}^{\mathrm{M}}$ (rows). Each tuple $l$ contains a group of cells as $\mathrm{C}_{l}=\left\{l\left[\mathrm{a}_{1}\right], l\left[\mathrm{a}_{2}\right], \ldots l\left[\mathrm{a}_{\mathrm{N}}\right]\right\}$ where $\mathrm{C}_{l}$ represents the cells in $l$, and $l\left[\mathrm{a}_{\mathrm{i}}\right]$ denotes the value of the $\mathrm{i}^{\text {th }}$ attribute in $l$. Also, $\mathrm{C}_{l} \subset \mathrm{C}$, where $\mathrm{C}=\left\{\mathrm{c}_{\mathrm{i}}\right\}_{\mathrm{i}=1}^{\mathrm{N} \times M}$ designates all the cells in a given $\mathrm{D}$. Given the example in Figure 1, $\mathrm{A}$ is a set that contains all the columns names (e.g., $\mathrm{A}=\{$ "ID", "Airport Name", "City", "State" $\}$ ), while $\mathrm{L}$ represents the four tuples in the table as $\mathrm{L}=\left\{\mathrm{l}_{1}, \mathrm{l}_{2}, \mathrm{l}_{3}, \mathrm{l}_{4}\right\}$. Similarly, the table is represented by a group of 16 cells, which signifies the four tuples. For example, $\mathrm{C}_{3}$ is a set that includes all four cells in the third tuple $l_{3}$ as $C_{3}=\{$ “12992", "Adams Field", "Little Rock", "AR" $\}$.

Since erroneous entries originate from assigning incorrect values (including missing values) to table cells, we assume that each cell $c_{i} \in C$ has a correct value $\overline{\mathrm{v}_{\mathrm{c}}}$ and a current observed value $\mathrm{v}_{\mathrm{ci}}$. Then, for each cell $c_{i} \in \mathrm{C}$, a cell $c_{i}$ is said to be erroneous if $\overline{\mathrm{v}_{\mathrm{ci}}} \neq \mathrm{v}_{\mathrm{ci}}$. For example, in Figure 1, the cell $c_{3}$ that represents the city name in the first tuple $\mathrm{l}_{1}$ has $\overline{\mathrm{v}_{\mathrm{c} 3}}=$ "Bethel" whereas its current observed value $\mathrm{v}_{\mathrm{c} 3}=$ "Beathel".

The model employs a training dataset $\mathrm{D}_{\mathrm{t}}$ in the second phase. The training dataset is denoted as $\left\{\mathrm{x}_{\mathrm{i}}, \mathrm{y}_{\mathrm{i}}\right\}_{\mathrm{i}=1}^{\mathrm{K}}$, where $\mathrm{K}$ is the number of training examples (cells) provided to fine-tune the proposed model and $x_{i}$ depicts a set of features representing a given cell (a record) in $D_{t}$ as $\left\{c_{i}, v_{c i}, \overline{v_{c i}}\right\}$. The features include a reference to each cell $c_{i}$ where $\left\{c_{i}\right\}_{i=1}^{K} \subset C$, and $\overline{v_{c i}}, v_{c i}$ express the correct and observed values for $c_{i}$, respectively. Additionally, $y_{i} \in\{-1,1\}$ represents the output label as a binary flag of a given cell (i.e., correct or erroneous). Generally, given a database table D and a training dataset $D_{t}$, the goal of the proposed model is to classify each cell value in $C$ by assigning a label to denote $c_{i}$ having a correct value $\{1\}$ or an erroneous one $\{-1\}$.

\subsection{Model Design}

The model architecture uses bidirectional encoder representations [19] with Gaussian Error Linear Unit (GELU) activation functions [40]. The model uses the encoder architecture with multiple selfattention layers to capture the dependency relationships between the cells and seize the tuplelevel representation. The encoder transforms the input data into another structured sequence. The input is internally altered using attention mechanisms [19] and position-aware connected layers. In our implementation, the number of transformer blocks (layers) is denoted as $\mathrm{B}=6$, and the number of self-attention heads is $S=12$. The "multi-headed" attention is a mechanism proposed in the transformer architecture to improve the model's capability to focus on different positions. With self-attention heads, the attention calculations [19] are repeated within each head to help the model capture multiple representation subspaces since the representation matrix is calculated $\mathrm{S}$ times with different weight matrices.

Thus, in the first phase, the model applies a self-supervised learning task to model the data representation. To train the model, we propose a Masked Data Model pretraining objective in 


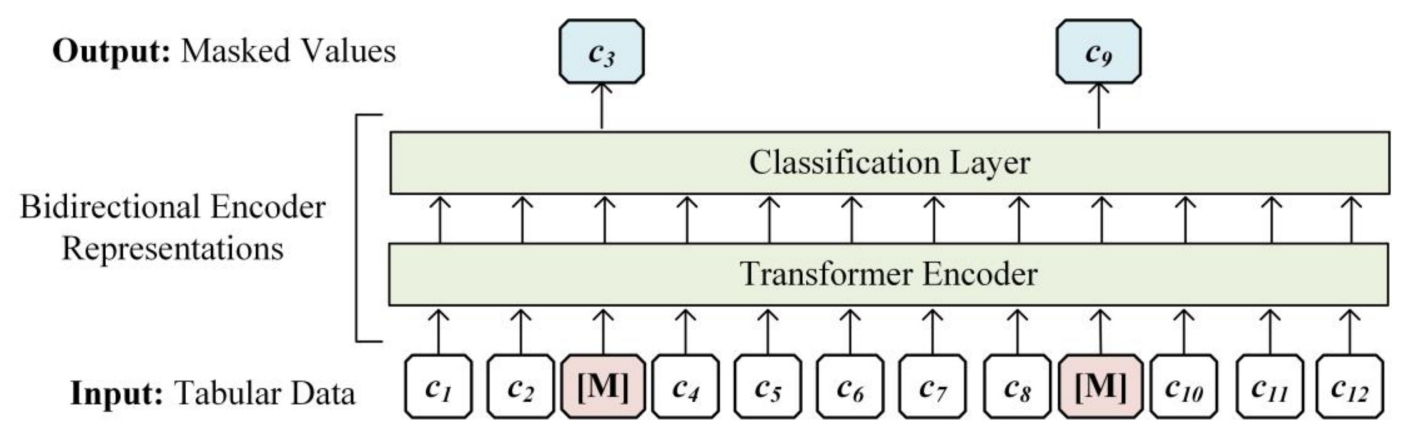

Fig. 3. Masked Data Model task in TabReformer.

which a fraction of the input cells is masked with a special token. An example of an MDM is shown in Figure 3. As the figure shows, the input tuple $C_{l}$ has 12 cells, with the cells $c_{3}$ and $c_{9}$ being replaced with a mask [M]. The model is trained to detect these cells. Then, for supervised finetuning, the model learns the task of erroneous data detection with the help of the labeled dataset $\mathrm{D}_{\mathrm{t}}$. To tackle the problem of imbalanced data, we introduce a data augmentation approach to generate more synthetic examples. In this stage, a generative process applies a set of transformations to the training examples in $\mathrm{D}_{\mathrm{t}}$. These transformations are executed on the correct values of each cell to create more erroneous values.

Although transformers are usually coupled with language modeling [23, 41-43], we show that using bidirectional transformer training can gain a deeper understanding of tabular data contexts. The following subsections offer further details for the implementation of TabReformer.

3.2.1 Bidirectional Transformers for Structured Data. For unsupervised pretraining, the proposed model operates on a sequence of values $\left\{l\left[\mathrm{a}_{1}\right], l\left[\mathrm{a}_{2}\right], . . l\left[\mathrm{a}_{\mathrm{N}}\right]\right\}$ representing the cells in a tuple l. Similar to Seq2seq models [41], the input sequence is processed by stacked encoder layers to output the encoded representation. However, to accommodate tabular data, we alter the structure of the first encoder input to process cells with continuous values without modification. Alternatively, cells with categorical values are mapped using trainable embeddings [45]. The model uses the feature_column.embedding_column function presented in TensorFlow to convert each value to single dimensional trainable embedding to handle categorical values. Furthermore, a trainable matrix is created for each categorical variable, in which each row represents a category. This embedding matrix is multiplied by the one-hot encoding of each cell value. Hence, categorical values are mapped to a single-dimensional trainable scalar and fed into the network. To accelerate the training phase, a preliminary step of instance normalization [46] is applied to standardize the input embeddings as

$$
E_{l\left[a_{i}\right]}^{\text {norm }}=\frac{E_{l\left[a_{i}\right]}-\mu_{i n}(E)}{\sqrt{\sigma_{i n}^{2}(E)+\epsilon}},
$$

where $E_{l\left[a_{i}\right]}^{n \text { norm }}$ is the normalized output of the input embedding $E_{l\left[a_{i}\right]}$ and $\mu_{i n}(E)$ and $\sigma_{i n}^{2}(E)$ are the instance means and variances computed as in [47]. The output of the normalization layer is then passed to an attentive transformer to model the dependencies between the attributes. Figure 4 shows an illustration of the transformer structure in the proposed framework. As the figure depicts, the input embeddings are passed to the first encoder. The output is then propagated to the following encoder layers as

$$
\mathrm{h}_{\mathrm{i}}=\operatorname{encoder}_{\text {block }\left(\mathrm{h}_{i-1}\right)}, \forall \mathrm{i} \in[1, \mathrm{~B}] .
$$




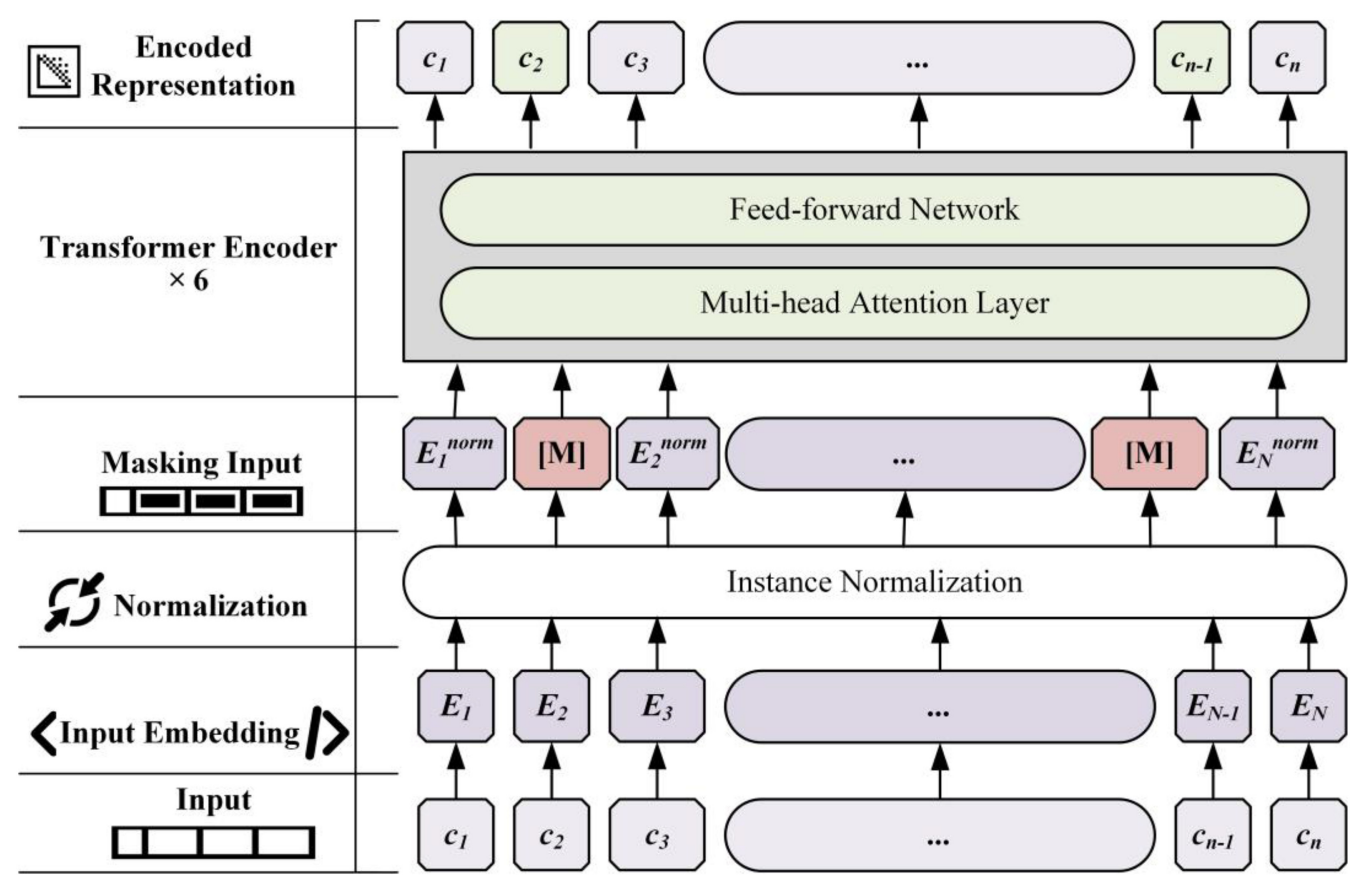

Fig. 4. Self-supervised learning in TabReformer.

Each encoder block consists of a multi-head attention layer followed by a layer of a feed-forward network. The multi-head attention layer applies, within each head, a set of transformations based on scaled dot product attention [19] to its input to capture the tuple-related features as

$$
\mathrm{Z}_{\mathrm{i}}=\operatorname{softmax}\left(\frac{Q_{i} \times K_{i}}{\sqrt{d_{k}}}\right) V_{i} \quad \forall \mathrm{i} \in[1, \mathrm{~S}],
$$

where $Q_{i}, K_{i}$, and $V_{i}$ are the query, key, and value matrices calculated for each head [19]. These matrices are multiplied together [19] to produce the representation matrices $Z$. These representations are then fed to a feed-forward neural network. As a self-supervised objective, we present the task of a masked data model. Similar to the Cloze task [23, 48], we mask 15\% of the cells in each instance at random, where each masked cell is replaced by a special symbol [M]. Then, the network is trained to predict the masked cells. To formalize the objective function, we use the log-likelihood as follows:

$$
L_{1}\left(C_{l} ; \theta\right)=\frac{1}{\left|C_{l}\right|} \sum_{c \in C_{l}} \log P\left(c_{i} \mid c_{\neq i} ; \theta\right),
$$

where $C_{l}=\left\{l\left[\mathrm{a}_{1}\right], l\left[\mathrm{a}_{2}\right], \ldots l\left[\mathrm{a}_{\mathrm{N}}\right]\right\}$ contains all of the values of the cells in $l, \mathrm{P}$ is the conditional probability that is modeled using the network with parameters $\theta$, and $c_{\neq i}$ denotes the values of the cells that appear before and after position $i$. In other words, the model aims to predict the masked token, given the instance inputs appearing before and after that token, and calculates the loss function for the masked data model. Generally, the task of detecting masked cells is analogous to identifying erroneous data. Since detecting errors in structured data requires extracting intercolumn relationships, bidirectional encoder representations can retrieve such relations. The transformer architecture forces the encoder to examine other cells in the input tuple as it 
generates the representation of a specific cell. As a result, the model can easily detect errors in cells that violate integrity constraints and functional dependencies, such as the example in Figure 1.

Thus, the objective allows us to obtain a bidirectional trained model with unsupervised representation. However, the mask tokens used in training may not appear during fine-tuning. Therefore, inspired by best practice found in the literature [23, 43], we replace $80 \%$ of the masked cells with a masked token, $10 \%$ are left unchanged, and $10 \%$ are swapped with values from other tuples.

Nevertheless, unsupervised representation learning in language modeling pretrained systems [23] usually includes multiple learning objectives. For example, the implementation of BERT [23] contains a Next Sentence Prediction (NSP) objective to train the model to infer the relationship between two sentences. For NSP, the model is pretrained with pairs of sentences, and the goal is to predict whether a given pair represents two consecutive sentences. Various implementations of transformer models $[49,50]$ have employed the next sentence prediction objective to different applications, other than language understanding, such as location prediction [49] and cross-modality matching [51]. Therefore, the experimental evaluation (Section 4.4) includes a sensitivity analysis to estimate the effectiveness of the next tuple prediction objective to the overall performance of TabReformer. However, we found that the next tuple prediction objective does not yield performance gains for erroneous data detection in tabular data. Also, previous studies [43] stated that removing the next sentence prediction loss can either match or improve the overall performance. Therefore, we have decided not to accommodate the next tuple prediction objective while training TabReformer.

3.2.2 Parameter Fine-tuning to Erroneous Data Detection. After training the model, the second phase fine-tunes the system parameters to the target task of erroneous data detection. Therefore, $\left\{\mathbf{x}_{\mathrm{i}}\right\}_{\mathrm{i}=1}^{\mathrm{K}}$ in $\mathrm{D}_{\mathrm{t}}$ are supplied as inputs, and the ground-truth labels $\left\{\mathbf{y}_{\mathrm{i}}\right\}_{\mathrm{i}=1}^{\mathrm{K}}$ are entered into an output layer for erroneous cell classification. To accomplish this task, the activation function of the final transformer block $h_{B}$ goes through a linear adder layer to predict y as

$$
\mathrm{P}\left(\mathrm{y} \mid \mathrm{x}_{\mathrm{i}}\right)=\operatorname{softmax}\left(\mathrm{h}_{\mathrm{B}} \mathrm{W}_{\mathrm{y}}\right),
$$

where $\mathrm{h}_{\mathrm{B}}$ is the output of the last encoder block and $W_{y}$ are the parameters of the linear output layer. Overall, the model aims to maximize the following objective function:

$$
\mathrm{L}_{2}(\mathbf{x})=\sum_{(\mathrm{x}, \mathrm{y}) \in \mathrm{D}_{\mathrm{t}}} \log \mathrm{P}(\mathrm{y} \mid \mathbf{x}) .
$$

To minimize the computational complexity, most of the model hyperparameters are kept identical except for $W_{y}$, the learning rate, and the number of training epochs. As mentioned before, the labeled dataset $D_{t}$ is utilized in this step for supervised fine-tuning. Given the fact that errors in datasets are often limited compared with the number of correct cells, the collected data is usually highly imbalanced. Since learning models tend to treat the minority class as noise and ignore it, this can affect the classification performance. To mitigate the imbalance risk, we execute a preliminary stage of data augmentation before fine-tuning the model. During such a step, more synthetic labeled points are generated with minimal manual effort. A detailed description of this stage is explained next.

3.2.3 Data Augmentation for Tabular Data. Since training neural networks with small unbalanced datasets can lead to overfitting, we employ a process of data augmentation to add more erroneous data points to $D_{t}$ before the fine-tuning phase. For this purpose, we aim to specify a set of transformation functions $\mathrm{T}$ that, when applied to correct values, can generate erroneous ones. Moreover, to decide on which transformations should be used to each cell value, we need to derive 
a strategy of error generation $S_{E G}$ [35]. Once both $\mathrm{T}$ and $S_{E G}$ are specified, the model can start learning additional training examples from $\mathrm{D}_{\mathrm{t}}$.

The stage begins by applying pattern matching to determine T. The only input to this phase is a set of training examples with no extra input required from end-users. Most state-of-the-art techniques, such as HoloDetect [15] and ED2 [52] require similar sets of labeled examples. Each training example includes the correct value of a specific cell and the observed value in this cell. The data augmentation phase in the proposed approach aims to generate more synthetic examples from the provided examples. Hence, to generate $\mathrm{T}$, the approach iteratively examines each of the labeled examples $\left\{c_{i}, \mathrm{v}_{\mathrm{ci}}, \overline{\mathrm{v}_{\mathrm{c}}} \mathrm{y}_{\mathrm{i}}\right\}$ and extracts all possible transformations $f \in \mathrm{T}$ that could be applied to $\overline{\mathrm{v}_{\mathrm{ci}}}$ to produce the observed value $\mathrm{v}_{\mathrm{ci}}$, so that $\mathrm{v}_{\mathrm{ci}}=f\left(\overline{\mathrm{v}_{\mathrm{ci}}}\right)$. During this iterative process, the model extracts a set of transformations from each erroneous example $D_{t}$ that represent how the errors are commonly introduced into the database.

A detailed explanation of the extraction process is presented in Algorithm 1. As the algorithm shows, the learning process applies the Gestalt Pattern Matching algorithm [53] to find the similarity between $v_{c i}$ and $\overline{v_{c i}}$ in each erroneous example in $D_{t}$ (lines $4-6$ ). The model returns the longest common substring found in $\mathrm{v}_{\mathrm{ci}}$ and $\overline{\mathrm{v}_{\mathrm{c}}}$. Then, it recursively returns the matching characters in the nonmatching regions on both sides of that substring (lines 7-13). The model then extracts the transformations by examining the overlap between the matching substrings and fits them with available operations. Finally, the algorithm merges all the sets derived from each example to produce a final multiset $\mathrm{F}$.

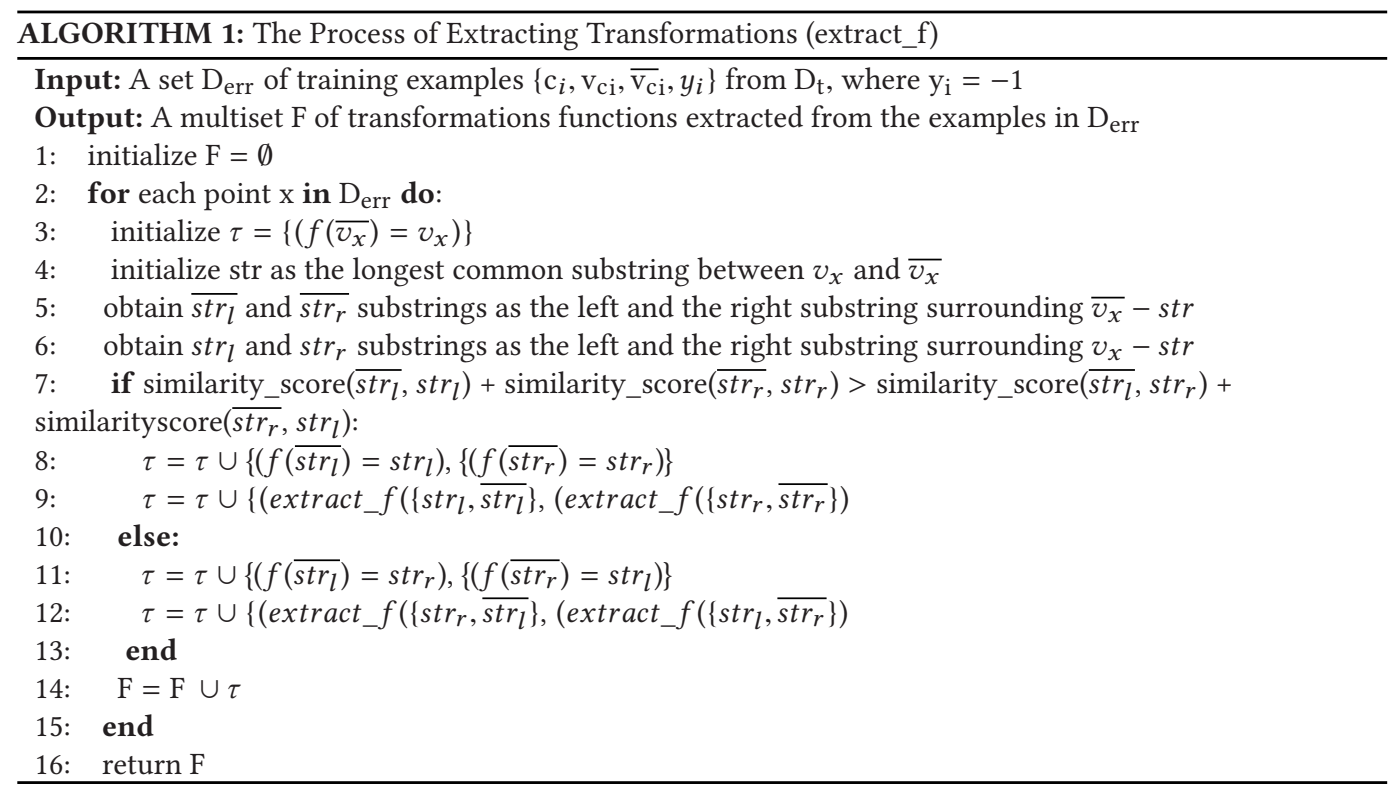

The process still needs to learn the strategy of error generation $S_{E G}$, which corresponds to the conditional probability distribution $\mathrm{P}\left(\mathrm{T} \mid \overline{\mathrm{v}_{\mathrm{c}}}\right)$ for a given correct value $\overline{\mathrm{v}_{\mathrm{ci}}}$ in $\mathrm{D}_{\mathrm{t}}$. Therefore, given the extracted multiset $\mathrm{F}$ from Algorithm 1 (line 14), the transformations set $\mathrm{T}$ is first constructed by removing the duplicated records in F. Then, the model learns $S_{E G}$ by first calculating the empirical distribution of each function in F. Since F is expected to have duplicated transformation functions applied to different data points, the empirical distribution of each transformation function $\mathrm{f}$ in $\mathrm{T}$ 
can be formally denoted as

$$
P(f)=\frac{\sum_{\mathrm{x} \in \mathrm{F}} 1\{\mathrm{x}=f\}}{|F|},
$$

where $|F|$ is the cardinality of $\mathrm{F}$, and $\sum_{\mathrm{x} \in \mathrm{F}} 1\{\mathrm{x}=f\}$ returns the number of times a function $f$ appears in F. The process is further explained in Algorithm 2.

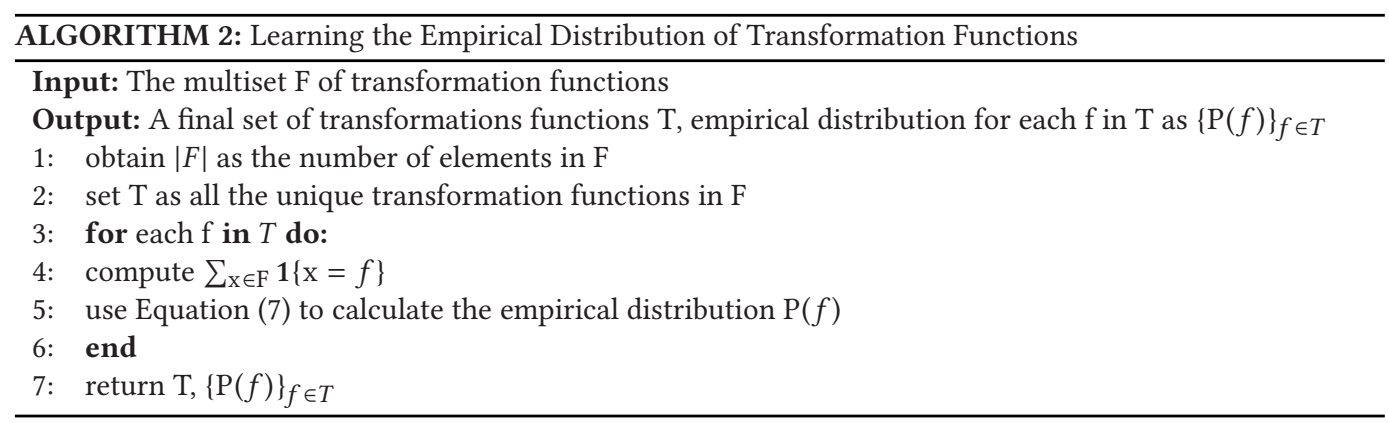

Then, to derive the conditional probability distribution $\mathrm{P}\left(\mathrm{T} \mid \overline{\mathrm{v}_{\mathrm{ci}}}\right)$ given a correct cell value $\overline{\mathrm{v}_{\mathrm{ci}}}$, the model finds all transformation functions in $\mathrm{T}$ as $f(\overline{s t r})=s t r$, such that str can be seen as a subset of $\overline{\mathrm{v}_{\mathrm{ci}}}$. Next, we consider the maximum and minimum of the empirical probabilities of these functions to normalize the empirical probability $\mathrm{P}(f)_{f \in T}$. The conditional probability can be formally denoted as

$$
\mathrm{P}\left(f \mid \overline{\mathrm{v}_{\mathrm{ci}}}\right)=\frac{\mathrm{P}(f)-\min \left(P\left(f_{\overline{\bar{c}_{i}}}\right)_{f \in T}\right)}{\text { norm }},
$$

where $f_{\overline{\mathrm{v}_{\mathrm{c}}}}$ is any transformation function over a substring of $\overline{\mathrm{v}_{\mathrm{ci}}}$ and norm is calculated as

$$
\text { norm }=\max \left(\mathrm{P}\left(\mathrm{f}_{\overline{\mathrm{v}_{\mathrm{c}}}}\right)_{\mathrm{f} \in \mathrm{T}}\right)-\min \left(\mathrm{P}\left(\mathrm{f}_{\overline{\mathrm{v}_{\mathrm{c}}}}\right)_{\mathrm{f} \in \mathrm{T}}\right) \text {. }
$$

These normalized empirical probabilities can be used by $S_{E G}$ to select which $f$ should be applied to a given value $\overline{\mathrm{v}_{\mathrm{c}}}$.

Consequently, the model randomly selects an instance from the correct training examples in $\mathrm{D}_{\mathrm{t}}$. Then, for each sampled data point, the conditional distribution $\mathrm{P}\left(\mathrm{T} \mid \overline{\mathrm{v}_{\mathrm{ci}}}\right)$ along with the learned transformations $\mathrm{T}$ are utilized to select appropriate transformation functions and to add more training examples to $\mathrm{D}_{\mathrm{t}}$. The new noisy value $\widetilde{\mathrm{v}_{\mathrm{ci}}}=f\left(\overline{\mathrm{v}_{\mathrm{ci}}}\right)$ is then added to $\mathrm{D}_{\mathrm{t}}$ as $\mathrm{D}_{\mathrm{t}}=\mathrm{D}_{\mathrm{T}} \cup\left\{c_{i}, \widetilde{\mathrm{v}_{\mathrm{ci}}}, \overline{\mathrm{v}_{\mathrm{ci}}}\right\}$. The algorithm takes a hyperparameter $\gamma$, which specifies the target ratio between correct and erroneous examples in the final training data $\mathrm{D}_{\mathrm{t}}$. During the experiments, the value of $\gamma$ is determined with cross-validation using a held-out set taken from $\mathrm{D}_{\mathrm{t}}$. The system also requires some design parameters, including the number of transformer blocks (B) and selfattention heads (S). However, changing these parameters affects the computational complexity of the system since the data has to travel through each block before calculating the final matrix of dimensions that describes the data representation. Also, the number of self-attention heads controls the number of different relationships between the inputs. These hyperparameters are set based on the insights obtained during the experimental evaluation (Section 4). Therefore, these numbers are considered design choices that define the model architecture. Typically, such parameters are set by machine learning engineers and can be altered to experiment with other arrangements.

As for the computational complexity, the transformer networks are proven to be more computationally effective [19] than other neural networks such as RNN. Since sequential operations in transformer networks are independent and can run in parallel, these operations in the proposed model can learn faster on parallel processing hardware. Hence, the computational complexity of 
the sequential operations is $\mathrm{O}(1)$ for self-attention layers. Formally, the complexity of each layer of the proposed method is $O\left(A^{2} \times d\right)$, where $\mathrm{A}$ is the number of attributes in each row and $\mathrm{d}$ is the vector dimension.

\section{EXPERIMENTAL EVALUATION}

The section presents empirical results obtained when comparing TabReformer against state-ofthe-art alternatives on a variety of real-world datasets. The experimental evaluation seeks to validate the following claims:

- Training a bidirectional transformer on structured data and fine-tuning it to the task of erroneous data detection can yield high-quality classification models when compared to existing error detection techniques. We compare TabReformer to other error detection techniques that rely on machine learning $[3,16,17,54]$. The experimental results show that the proposed method outperforms other deep learning methods [3] by $45.86 \%$ on average (recall). Also, the experiments illustrate that the final trained model improves precision by $16.90 \%$ and the recall by $29.28 \%$ on average when compared to other machine learning techniques [16, 17, 54].

- Data augmentation represents an optimal approach for obtaining ample training data while minimizing the required human effort. We compare the data augmentation module in TabReformer to other paradigms for collecting training data such as supervised learning and active learning [16]. Along with performance metrics, the experiments consider user effort to evaluate the model. The experimental results show that the proposed method can enhance the classification performance by $28.69 \%$ on average (F1 measure) while reducing the manual labeling effort by up to $48.86 \%$.

In addition, we perform a micro-benchmark to evaluate the individual design choices of TabReformer, such as the effectiveness of data augmentation and adding other training objectives. Finally, we conduct a sensitivity analysis to evaluate TabReformer with different error rates and test how it handles mildly to very dirty data.

The section is divided into six subsections. In the first subsection, we discuss the datasets and the baseline techniques, along with the evaluation setup. Next, we report the results of comparing the proposed method to other error detection methods [3, 16, 17, 54]. Then, to validate the data augmentation claim, we compare data augmentation to traditional active learning and experiment with different values for the labeling cost. In the fourth subsection, we evaluate the individual components of the proposed system by experimenting with two variations of our model, in which we investigate different design choices and learning paradigms. A sensitivity analysis is presented (in the fifth subsection), in which the experiments are repeated with different ranges of error types and ratios. The analysis aims to evaluate the effectiveness of the proposed technique with different experimental parameters, such as error rates and types. Finally, the sixth section introduces a general discussion, along with suggestions for future directions

\subsection{Evaluation Setup}

Datasets: The experiments utilize six datasets that explore a wide range of domains and vary in size, dimensionality, error types, and distributions. The summary statistics of these datasets are provided in Table 1. The table shows, for each dataset, the number of tuples (Size), the number of columns (Dimensionality), the initial size of training data $D_{t}(K)$, the number of erroneous cells (Errors (\# of cells)), the corresponding percentage of incorrect cells divided by the total number of cells in each dataset (Errors \%), and error types injected into each dataset. Although existing research $[15,16]$ has experimented with low ratios of injected errors (less than $2.5 \%$ ), recent 
Table 1. Datasets used in the Evaluation ${ }^{\mathrm{a}}$

\begin{tabular}{lcccccc}
\hline Dataset & Size & Dim. & \multicolumn{1}{c}{ K } & Errors (\# of cells) & Errors \% & Error Types \\
\hline Adult & 48,842 & 14 & 2,100 & 72384 & 12.30 & T, VS \\
Restaurants & 28,788 & 16 & 1,439 & 19168 & 14.40 & T,VS,VD \\
Flights & 13,884 & 10 & 819 & 7297 & 13.10 & T, FE,VD \\
Movies & 7,390 & 17 & 318 & 14193 & 13.60 & T,VS \\
Hospital & 4,561 & 19 & 283 & 2480 & 13.50 & T, MV, VS \\
Beers & 2,410 & 11 & 147 & 3152 & 11.80 & T \\
\hline
\end{tabular}

Dim. refers to the number of columns, $\mathrm{K}$ is the size of training data $\mathrm{D}_{\mathrm{t}}$, and error types are typographical errors (T), value swapped across columns (VS), values violating data constraints (VD), formatting errors (FE), and missing values (MV).

surveys $[55,56]$ show that real-world datasets contain higher percentages of inaccurate entries and data errors (more than 10\%) [55]. Therefore, the experiments consider the ratios reported in these surveys to set up more elevated injected error rates.

The first dataset used in the experiments is the Adult dataset, which is a benchmark dataset [57] collected by Barry Becker from the 1994 Census database and is used to evaluate other detection and repairing techniques $[15,16]$. The dataset contains various attributes for individuals, such as their education level, age, gender, and annual income. The second dataset in the experiments is the Restaurant dataset, which contains information about restaurants in the United States. The experiments also consider the Flights dataset [22], which comprises departure and arrival information on domestic flights in the United States. The data is collected by the U.S. Bureau of Transportation Statistics and is used to evaluate other detection approaches, such as Raha [54] and HoloClean [3].

Similarly, Movies and Beers are two real-world datasets that have been collected from recent detection techniques [54,52]. The Movies dataset includes information about movies crawled from IMDB, while the Beers dataset encompasses different beer styles and brands. The Beers data was crawled from CraftCans.com in 2017 and contains missing values, value swaps across tuples, and typographical errors. Finally, the experiments include the Hospital dataset, which is also a benchmark dataset used to evaluate several error detection tools $[2,15]$.

Competing methods: We compare TabReformer against the following baseline techniques:

- HoloClean [3] is a state-of-the-art holistic error detection and data repairing technique driven by probabilistic inference. The current implementation of HoloClean applies various types of error detection methods, which include denial constraints violation [11], outlier detection [7], and missing values detection. Then, the system unifies all the outputs of these methods by utilizing probability theory. It tries to estimate a hidden clean version of the dataset by utilizing each output as evidence on the accuracy of the records of the dataset. The experiments evaluate only the detection capabilities of HoloClean since data repairing is beyond the scope of this article.

-ED2 [16] is a two-stage, example-driven error detection method. The method first applies a classification strategy to choose the cells that need to be tagged by the end-user as correct or erroneous. After collecting labeled data from the user, the method utilizes a wide range of features to represent the data and detect incorrect cells. When applied to datasets with relatively small error ratios, the model reports superior performance over the state-of-theart solutions $[9,15]$ while reducing the effort of manual labeling.

- Raha [54] is an error detection approach that utilizes different error detection algorithms and configurations to generate feature representations for the data. The method also applies 
a clustering algorithm and label propagation technique to optimize the labeling efforts required to provide training labels.

- ActiveClean [17] is an iterative data cleaning tool that applies statistical model training to detect erroneous data cells recursively. The approach employs a selection of convex loss models to clean dirty data and improve classification performance iteratively. ActiveClean applies a sampling algorithm that selects data batches that need to be cleaned by end-users. It then feeds this clean data into the model to retrain it and recommend other batches to the user.

- NADEEF [9] is another error detection and data cleansing framework that allows users to define data quality rules that specify data problems using a programming interface. NADEEF compiles all of these rules and examines the data to select violating cells.

Other error detection techniques are recently proposed, however, such as HoloDetect [15] and Uni-Detect [58]. HoloDetect [15] uses few-shot learning to build a neural network for erroneous data detection. The system integrates weak supervision with supervised learning to leverage noisy signals from data models and train machine learning models. Like the proposed technique, it applies data augmentation in a weakly supervised setting to obtain enough training labels. Also, UniDetect is a unified framework that employs principled reasoning of errors using hypothesis-tests. The method analyzes a large corpus of tables crawled from the Web. Then, the method utilizes the analysis results to detect errors for any new dataset. Unfortunately, since no available implementations for these approaches $[15,58]$ could be found, we could not compare TabReformer to these systems. Nonetheless, we include some of the datasets that are used in their experiments in our experimental evaluation.

Variants of TabReformer: We also experiment with the following three variants of TabReformer.

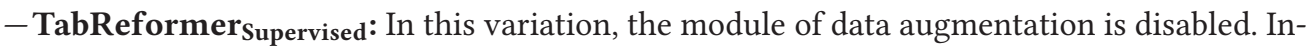
stead, the model is fine-tuned using the initial data points provided in $\mathrm{D}_{\mathrm{t}}$.

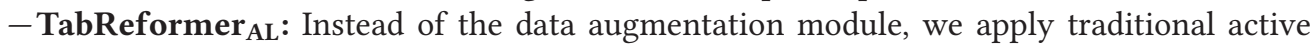
learning [59] to obtain additional training examples. First, the model is fine-tuned with $D_{t}$. Then, we employ uncertainty sampling for $r$ iterations. During every iteration, the user is queried to label a batch of 50 examples. Then, the model is retrained and evaluated.

- TabReformer $_{\mathrm{NTP}}$ : In this version, the training phase is repeated with the next tuple prediction (NTP) objective enabled. Similar to the state-of-the-art models of natural language processing [23, 43], we train the model with pairs of tuples as input to predict whether the second tuple follows the first one in the input table $\mathrm{D}$. The training data has $50 \%$ of the input as consecutive tuples (with label $y=1$ ), while the rest are separate tuples chosen randomly from $\mathrm{D}$ (with label $\mathrm{y}=-1$ ). The training loss, in this case, is the sum of the mean masked data model likelihood and the mean next tuple prediction likelihood. In the literature [23, 44], the next sentence prediction helps the model to understand sentence-level representation. Therefore, we add this version of the model to investigate whether this will improve the model's capability to capture table-level contexts.

Experimental Setup: To measure the effectiveness of error detection, we report Precision (P), Recall (R), and F1 measures. All datasets used in the experiments have clean versions that are used as the ground truth. During the training phase, the existing ground truth is split to form the labeled training data $D_{t}$, an unlabeled pool for the active learning experiments, a test set for evaluation, and a held-out set for hyperparameter tuning. To optimize TabReformer, we use ADAM [60] with a learning rate of 0.02 . 
Table 2. Evaluation Metrics of Different Methods for Error Detection

\begin{tabular}{cccccccc}
\hline $\begin{array}{c}\text { Dataset } \\
\left(\text { Size of } \mathrm{D}_{\mathrm{t}}\right)\end{array}$ & $\begin{array}{c}\text { Evaluation } \\
\text { Metric }\end{array}$ & TabReformer & HoloClean & ED2 & Raha & ActiveClean & NADEEF \\
\hline Adult & $\mathrm{P}$ & $\mathbf{0 . 9 7}$ & 0.81 & 0.90 & 0.71 & 0.92 & 0.90 \\
$(4.30 \%)$ & $\mathrm{R}$ & $\mathbf{0 . 9 2}$ & 0.62 & 0.87 & 0.55 & 0.60 & 0.91 \\
& $\mathrm{~F}_{1}$ & $\mathbf{0 . 9 4}$ & 0.70 & 0.88 & 0.62 & 0.73 & 0.90 \\
\hline Restaurant & $\mathrm{P}$ & 0.81 & 0.71 & 0.77 & 0.63 & $\mathbf{0 . 8 3}$ & 0.81 \\
$(5.00 \%)$ & $\mathrm{R}$ & $\mathbf{0 . 9 6}$ & 0.65 & 0.91 & 0.60 & 0.52 & 0.70 \\
& $\mathrm{~F}_{1}$ & $\mathbf{0 . 8 8}$ & 0.68 & 0.83 & 0.61 & 0.64 & 0.76 \\
\hline Flights & $\mathrm{P}$ & $\mathbf{0 . 9 6}$ & 0.82 & 0.74 & 0.84 & 0.66 & 0.79 \\
$(5.90 \%)$ & $\mathrm{R}$ & $\mathbf{0 . 9 1}$ & 0.67 & 0.90 & 0.51 & 0.61 & 0.69 \\
& $\mathrm{~F} 1$ & $\mathbf{0 . 9 3}$ & 0.74 & 0.81 & 0.63 & 0.63 & 0.74 \\
\hline Movies & $\mathrm{P}$ & $\mathbf{0 . 9 7}$ & 0.72 & 0.88 & 0.33 & 0.79 & 0.90 \\
$(4.30 \%)$ & $\mathrm{R}$ & $\mathbf{0 . 9 4}$ & 0.60 & 0.60 & 0.80 & 0.64 & 0.52 \\
& $\mathrm{~F} 1$ & $\mathbf{0 . 9 5}$ & 0.65 & 0.71 & 0.47 & 0.71 & 0.66 \\
\hline Hospital & $\mathrm{P}$ & 0.92 & 0.93 & 0.80 & $\mathbf{1 . 0 0}$ & 0.91 & 0.87 \\
$(6.20 \%)$ & $\mathrm{R}$ & $\mathbf{0 . 8 1}$ & 0.64 & 0.79 & 0.72 & 0.69 & 0.70 \\
& $\mathrm{~F} 1$ & $\mathbf{0 . 8 6}$ & 0.76 & 0.79 & 0.84 & 0.78 & 0.78 \\
\hline Beers & $\mathrm{P}$ & $\mathbf{0 . 9 6}$ & 0.84 & 0.93 & 0.65 & 0.59 & 0.91 \\
$(6.10 \%)$ & $\mathrm{R}$ & $\mathbf{0 . 9 0}$ & 0.70 & 0.84 & 0.84 & 0.60 & 0.87 \\
& $\mathrm{~F} 1$ & $\mathbf{0 . 9 3}$ & 0.76 & 0.88 & 0.73 & 0.59 & 0.89 \\
\hline
\end{tabular}

In the experiments, we used ED2 with the min certainty [16] as the column selection strategy and a learning batch size of 50 cells. The labeling cost consumed by ED2 is set to $4 \%$ of the total size of each dataset. The limit is selected since it is reported as the optimal cost in their experimental evaluation [16]. As for ActiveClean, the model is initially trained using $D_{t}$. Then, in each iteration, ActiveClean recommends a batch of 50 tuples to be cleaned by the user. After that, the approach updates (retrains) the current model using the obtained clean data and selects the next batch. This iterative clean-retrain process is repeated until an optimal clean model is realized [17]. According to their experiments, the labeling budget is usually set to be around $2 \%$ to $10 \%$. Therefore, our experimental evaluation considers a maximum number of iterations as $i / 50$, where $i=6 \%$ of the size of each dataset. Section 4.3 represents additional experiments to assess the sensitivity of the cost variable. Additionally, to evaluate the methods that rely on data sampling, such as ED2 and TabReformer $_{\mathrm{AL}}$, we repeated the experiments ten times and reported the arithmetic mean.

\subsection{End-to-End Performance}

In this section, we compare the classification performance of TabReformer to detect data errors against the competing approaches in the six datasets. The experimental results achieved by different methods are presented in Table 2. For each method, the table shows the precision, recall, and F1 measures. These values represent the average (arithmetic mean) of 15 runs. The table also illustrates the percentage of training data $D_{t}$ of the total dataset. These percentages refer to the initial size of the training data before applying the data augmentation module (see Section 3.2.3).

As Table 2 illustrates, TabReformer consistently achieves better F1 measure performance than other approaches in all of the datasets. For example, in the Movies and Flights datasets, TabReformer can enhance the performance (F1 measure) by $52.91 \%$ and $32.63 \%$, respectively. Also, in most of the datasets, TabReformer attains the highest precision and recall values, especially in 
the datasets that contain multiple types of errors with different distributions. For instance, the results show that in the Restaurants dataset, the proposed approach outperforms HoloClean in precision and recall values by $13.87 \%$ and $47.39 \%$, respectively. Since the performance of HoloClean highly depends on the quality of its error detection techniques, it shows imperfect results in datasets, which include several error types. On average, the results depict that TabReformer could improve the precision and recall values by $22.18 \%$ and $34.55 \%$, respectively, when compared to the other techniques.

Alternatively, the results show that NADEEF consistently achieves reasonable precision values in most datasets since it can detect cells violating predefined quality rules. However, it fails to report other types of errors, such as value swaps and typos, which usually result in a significantly low recall (e.g., 0.52 in the Movies dataset). Additionally, in most of the datasets, results illustrate that data representation plays an essential role in detecting various types of errors. For example, in the Flights dataset, most errors come from cells violating integrity constraints and functional dependencies (Figure 1). Hence, modeling the data can substantially enhance classification performance in these tasks.

Consequently, approaches that depend on learning the data representation, such as TabReformer and ED2, manage to achieve higher recall in these situations. For instance, TabReformer attains better recall in the Flight dataset by a maximum improvement of $78.43 \%$ compared to Raha. Similarly, ED2 outperforms HoloClean in the same dataset in recall values. However, when dealing with a higher volume of errors, such as in the Movies and Hospital datasets, ED2 reports poor recall values due to the diversity of error distributions and the limited labeling effort.

Also, Raha shows adequate precision values in most of the datasets. Since it integrates the output from many error detection strategies, it reports different types of errors. For example, in the Hospital and Flight datasets, Raha manages to outperform most competing tools with a maximum improvement of $27.27 \%$ compared to ActiveClean. However, Raha reports low precision value (0.33) in the Movies dataset due to the large number of value swap errors injected in the dataset, which cannot be detected by the underlying detection tools. Similarly, in datasets with high numbers of value swaps, Raha achieves lower recall values than the other techniques. For example, in the Adult dataset, Raha was outperformed by all of the other tools, as it achieved the worst recall value (0.55).

As for ActiveClean, the evaluation shows that it fails to capture the necessary tuple-level characterization to classify erroneous cells. For example, in the Restaurant dataset, ActiveClean reports the worst F1 measure due to its significantly lower recall (0.52), as it fails to detect any of the typographical errors. Moreover, in the Flights dataset, the tool reports many false positives, which results in the lowest value of precision (0.61) among the competing techniques. Accordingly, we postulate that these results agree with our assumption that modeling data characteristics improve the classification of erroneous cells. Overall, the results empirically posit that, since TabReformer uses a bidirectional transformer to model the data, it manages to output more accurate results and detect a broader range of error types with different error distributions.

The results demonstrate that HoloClean shows an adequate performance in datasets with outliers, missing values, and constraints violations. For example, HoloClean enhances the precision values in the Hospital dataset by $16.25 \%$ and $6.90 \%$ compared to ED2 and NADEEF, respectively. However, in the Movies and Flights datasets, HoloClean reports low recall values ( 0.60 and 0.67 , respectively). Most of the reported errors are related to integrity constraints defined for these datasets. Nevertheless, since most of the errors injected in these datasets require exploiting intercolumn relationships and functional dependencies, the performance of HoloClean was bounded by detecting cells violating denial constraints. 


\subsection{Data Augmentation Versus Active Learning}

To estimate the effectiveness of data augmentation, we compare it to traditional active learning and study the impact of the labeling cost to the performance of the competing methods. Since neural networks require a large amount of labeled data, active learning has been used to alleviate this obstacle by selecting a small number of unlabeled samples. Traditional active learning strategies, especially uncertainty sampling, have been used in many use cases such as information extraction [27] and machine translation [61]. Hence, we first validate the claim that data augmentation can optimize labeling effort while achieving a satisfactory performance for erroneous values classification. Therefore, we train a new model TabReformer $\mathrm{AL}$ in which we disable the data augmentation step and replace it with uncertainty sampling [59]. In this version, the model is first trained via unsupervised learning over D (see Section 3.2.1). Then, during fine-tuning, the model applies active learning with uncertainty sampling for several iterations. Uncertainty sampling ranks the output of the last layer to select the point about which the network is most uncertain. In each iteration, the model acquires labels for a batch of 50 examples of erroneous cells and adds this batch to $\mathrm{D}_{\mathrm{t}}$. After obtaining these labels, the fine-tuning phase is repeated and the model is evaluated using a test set. Finally, we compare the new model TabReformer ${ }_{\mathrm{AL}}$ against the original implementation of TabReformer to validate the claim of data augmentation.

To study the effect of labeling cost, the experiments with ED2 and ActiveClean are repeated with different numbers of iterations $r \in\{5,10,20,50\}$. The initial labeled data $D_{t}$ is set as in Table 1, and we report the F1 measure of each of the competing approaches with additional iterations. Also, since TabReformer does not utilize any labeled data from the user, the number of labeled examples obtained during each setup of $r$ is computed and added to $D_{t}$. Then, TabReformer utilizes this updated version of $\mathrm{D}_{\mathrm{t}}$ during the data augmentation module to generate more synthetic data points.

For each dataset, the F1 measure, for each of the four models (TabReformer, ED2, ActiveClean, and TabReformer $\mathrm{AL}_{\mathrm{AL}}$ ), is plotted against different $r$ values (Figure 5). As the figure shows, TabReformer attains better F1 scores in all the datasets. Extending $D_{t}$ with more examples allows TabReformer to achieve a higher F1 measure since the data augmentation algorithm can learn more training examples and represent different errors. For instance, in the Adult dataset, TabReformer initially attains an F1 measure value of 0.83 with a small number of iterations $(r=10)$. Subsequently, escalating $\mathrm{D}_{\mathrm{t}}$ with more iterations $(\mathrm{r}=20$ and $\mathrm{r}=50)$, improves the performance of TabReformer by $10.84 \%$ and $4.34 \%$, respectively.

Additionally, when comparing TabReformer with TabReformer ${ }_{\mathrm{AL}}$, the results illustrate that data augmentation outputs better models than active learning. For instance, in large datasets such as the Adult and Restaurant datasets, the data augmentation module improves the performance of the error detection by $15.28 \%$ and $8.01 \%$, respectively, when compared to TabReformer $\mathrm{AL}$ with ten iterations of active learning $(\mathrm{r}=10)$.

Also, the results suggest that, in many situations, ActiveClean shows a faster converge than ED2. For example, in the Flights dataset, ActiveClean consumed fewer iterations (with $r=20$ ) to reach global converge, while ED2 requires more than 40 iterations. In general, the results demonstrate that, since TabReformer applies data augmentation while modeling the data representation, the approach can realize high-quality data models with minimum manual efforts.

\subsection{Micro-Benchmarking}

To evaluate the effect of different design decisions implemented in TabReformer, we conduct an additional set of experiments to compare different variations of the system. First, to assess the training objective, we repeat the training stage with an additional objective function to predict the 


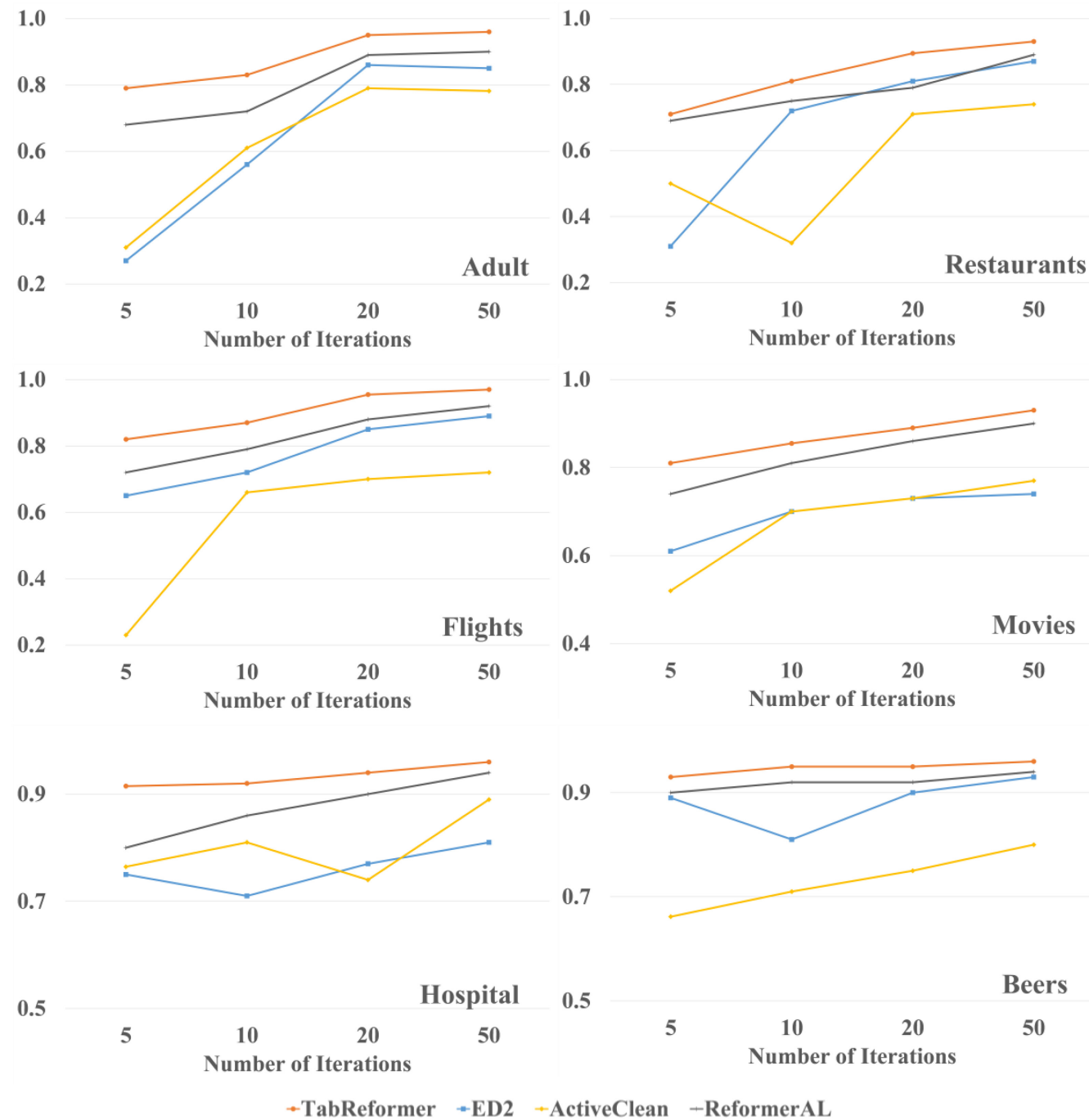

Fig. 5. F1-score of detection methods with increasing labeling efforts.

next tuple. The new model, TabReformer ${ }_{\mathrm{NTP}}$, combines the (arithmetic) mean of the two objective functions and uses it as the training loss. Second, we disable the data augmentation module, and the model is fine-tuned using the initial version of $D_{t}$. To assist with the problem of data imbalance, the new model TabReformer ${ }_{\text {Supervised }}$ resamples the points in $D_{t}$ to make sure that both classes are presented corresponding to the hyperparameter $\gamma$ [62]. We repeat the experiments in Section 4.2 to compare between the original implementation TabReformer and the two variations, TabReformer $_{\mathrm{NTP}}$ and TabReformer ${ }_{\text {Supervised, }}$ with differing sizes of training data $\mathrm{D}_{\mathrm{t}}$. The experimental results are summarized in Table 3. For each dataset, the table shows the values of Precision, Recall, and F1 measure achieved by each model while increasing the size of $\mathrm{D}_{\mathrm{t}}$.

The table shows that, even with different setups of $\mathrm{D}_{t}$, using data augmentation consistently results in higher-quality models. TabReformer outperforms the other two variations in all of the datasets. With large datasets such as the Adult and Restaurants datasets, TabReformer 
Table 3. Performance of TabReformer Supervised $_{\text {and TabReformer }}$ NTP with Increasing Sizes of Training Data

\begin{tabular}{|c|c|c|c|c|c|c|c|c|c|c|}
\hline \multirow[t]{2}{*}{ Dataset } & \multirow{2}{*}{$\begin{array}{c}\text { (Size of } \\
\left.D_{t}\right) \%\end{array}$} & \multicolumn{3}{|c|}{ TabReformer } & \multicolumn{3}{|c|}{ TabReformer ${ }_{\text {Supervised }}$} & \multicolumn{3}{|c|}{ TabReformer $_{\text {NTP }}$} \\
\hline & & $\mathrm{P}$ & $\mathrm{R}$ & F1 & $\mathrm{P}$ & $\mathrm{R}$ & F1 & $\mathrm{P}$ & $\mathrm{R}$ & F1 \\
\hline \multirow{3}{*}{ Adult } & $5 \%$ & 0.97 & 0.95 & 0.96 & 0.71 & 0.64 & 0.67 & 0.91 & 0.89 & 0.90 \\
\hline & $10 \%$ & 0.97 & 0.98 & 0.97 & 0.72 & 0.67 & 0.69 & 0.93 & 0.90 & 0.91 \\
\hline & $15 \%$ & 0.97 & 0.97 & 0.97 & 0.75 & 0.70 & 0.72 & 0.95 & 0.87 & 0.91 \\
\hline \multirow{3}{*}{ Restaurants } & $5 \%$ & 0.92 & 0.87 & 0.89 & 0.68 & 0.65 & 0.66 & 0.83 & 0.87 & 0.85 \\
\hline & $10 \%$ & 0.94 & 0.91 & 0.92 & 0.75 & 0.69 & 0.72 & 0.87 & 0.90 & 0.88 \\
\hline & $15 \%$ & 0.96 & 0.90 & 0.93 & 0.79 & 0.71 & 0.75 & 0.89 & 0.90 & 0.89 \\
\hline \multirow{3}{*}{ Flights } & $5 \%$ & 0.93 & 0.95 & 0.94 & 0.57 & 0.23 & 0.33 & 0.92 & 0.95 & 0.93 \\
\hline & $10 \%$ & 0.90 & 0.97 & 0.93 & 0.66 & 0.56 & 0.61 & 0.93 & 0.92 & 0.92 \\
\hline & $15 \%$ & 0.96 & 0.92 & 0.94 & 0.70 & 0.69 & 0.69 & 0.94 & 0.92 & 0.93 \\
\hline \multirow{3}{*}{ Movies } & $5 \%$ & 0.86 & 0.87 & 0.86 & 0.68 & 0.64 & 0.66 & 0.90 & 0.81 & 0.85 \\
\hline & $10 \%$ & 0.83 & 0.89 & 0.86 & 0.70 & 0.65 & 0.67 & 0.91 & 0.83 & 0.87 \\
\hline & $15 \%$ & 0.92 & 0.91 & 0.91 & 0.76 & 0.70 & 0.73 & 0.93 & 0.88 & 0.90 \\
\hline \multirow{3}{*}{ Hospital } & $5 \%$ & 0.93 & 0.91 & 0.92 & 0.58 & 0.55 & 0.56 & 0.87 & 0.83 & 0.85 \\
\hline & $10 \%$ & 0.92 & 0.96 & 0.94 & 0.67 & 0.59 & 0.63 & 0.91 & 0.87 & 0.89 \\
\hline & $15 \%$ & 0.95 & 0.95 & 0.95 & 0.77 & 0.68 & 0.72 & 0.90 & 0.93 & 0.91 \\
\hline \multirow{3}{*}{ Beers } & $5 \%$ & 0.96 & 0.90 & 0.93 & 0.51 & 0.41 & 0.45 & 0.89 & 0.91 & 0.90 \\
\hline & $10 \%$ & 0.95 & 0.94 & 0.94 & 0.55 & 0.72 & 0.62 & 0.90 & 0.89 & 0.89 \\
\hline & $15 \%$ & 0.98 & 0.96 & 0.97 & 0.62 & 0.76 & 0.68 & 0.93 & 0.94 & 0.93 \\
\hline
\end{tabular}

manages to enhance the F1 measure by $41.12 \%$ and $34.55 \%$, respectively, when compared to TabReformer ${ }_{\text {Supervised }}$ with the small size of $\mathrm{D}_{\mathrm{t}}(\mathrm{K}=5 \%)$. Since TabReformer Supervised $_{\mathrm{s}}$ relies on the examples provided in $\mathrm{D}_{\mathrm{t}}$, the model performance suffers from imbalanced data. Although resampling is applied to mitigate this risk, the error heterogeneity magnifies the impact of the imbalance problem. Thus, resampling cannot represent different error types in the training data, which results in poor performance of the supervised learning model.

Furthermore, in datasets with different error distributions, TabReformer Supervised $_{\text {produces un- }}$ satisfactory results. For instance, in the Flights dataset, the supervised version records a value of 0.33 for the F1 measure since it reports only $23.81 \%$ of the errors injected in the Flights dataset (Recall). Moreover, increasing the size of the training data does not seem to help with the imbalance problem. With bigger training data, TabReformer Supervised $_{\text {is also outperformed by the other }}$

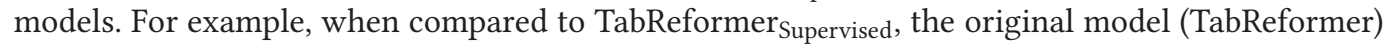
can enhance the detection quality by $31.54 \%$ (F1 measure) in the Hospital dataset when training the models with $15 \%$ of the dataset. Likewise, TabReformer ${ }_{\mathrm{NTP}}$ reports a $26.66 \%$ enhancement in the same dataset when compared to the supervised version. Overall, the empirical results confirm that data augmentation can form a reliable solution for alleviating different imbalance levels and varying ratios of errors.

Additionally, the table shows the results of comparing the original model with TabReformer ${ }_{\text {NTP }}$ and depicts that, in most cases, training the model with NTP loss does not yield any performance improvements. Instead, removing the NTP objective can slightly improve the overall performance, especially with large datasets. For example, the original implementation of TabReformer improves the classification performance in the Adult and the Restaurant datasets by $5.76 \%$ and $4.53 \%$ on average compared to TabReformer ${ }_{\text {NTP }}$ (F1 measure), respectively. Alternatively, adding the NTP loss results in approximately the same performance for many cases, such as the Flights and Movies 
datasets (e.g., the only improvement TabReformer ${ }_{\text {NTP }}$ achieved is recorded within the Movies dataset, with only $1.07 \%$ enhancement over the original implementation of TabReformer, with $\mathrm{D}=$ $10 \%)$. Generally, although training with next sentence prediction can enhance modeling unstructured text [23], the situation is different for tabular data. We find that dataset-related representation does not depend on the relationships between the tuples. Instead, deep bidirectional representation of data features can expand the model performance for error classification.

\subsection{Sensitivity Analysis}

To further evaluate the proposed method with different experimental parameters, a sensitivity analysis is conducted in which the experiments in Section 4.1 are repeated with various error rates and ratios. This section is divided into two subsections. In the first subsection, TabReformer, along with the baseline techniques, are evaluated with different error rates. In the second subsection, the performance of the proposed technique is tested with different permutations of the input attributes along with different ratios for specific error types.

4.5.1 Error Rate Impact. To analyze the impact of the error rates on the performance of TabReformer, we modified the datasets used in the experiments to inject different rates of erroneous cells. For each dataset, we replaced $2.5 \%, 5 \%, 10 \%, 20 \%$, and $40 \%$ of the cells in the datasets with erroneous values (e.g., missing values, cells violating data constraints, and values swapped across columns). Also, we repeated the experiments with each error rate ten times and reported the average (arithmetic mean) of the F1 measure achieved by each of the competing tools. The results are illustrated in Figure 6 and show that the proposed method can attain a higher F1 measure in all of the datasets with different error rates.

In most of the datasets, TabReformer can achieve significant improvements when dealing with small error rates. For example, with error rate $=2.5 \%$ (Errors $\%$ ), the proposed method outperforms the other tools with $43.66 \%$ and $31.59 \%$ in the Beers and the Flights datasets, respectively. Similarly, TabReformer surpasses the baseline techniques in all of the datasets with an error rate $=10 \%$, with a maximum improvement of $52.64 \%$ in the Movies dataset. Generally, a low rate of errors presents a challenge for classification models, since it requires increasing the training data to enhance the model capability to detect the minority class. Therefore, this empirically proves the effectiveness of the data augmentation module in TabReformer in alleviating the problem of training data scarcity. Alternatively, detection methods that rely on machine learning, such as ActiveClean, show decaying performance with lower error rates. For example, although ActiveClean could achieve an adequate performance level in the Hospital datasets with error rate $=20 \%$ (F1 measure $=87.18 \%$ ), its performance declined in the same dataset when the error rates decreased (Errors\% $=2.5 \%$ ) with more than a $31.03 \%$ reduction. Likewise, ED2 performance shows the same pattern with lower rates of errors in most of the datasets. In the Flights and the Beers datasets, the model performance dropped by $23.53 \%$ and $10.53 \%$ when the error rates declined from $20 \%$ to $2.5 \%$.

On the other hand, the other techniques that do not require training data, such as HoloClean, Raha, and NADEEF, show consistent performance for different error rates. For example, ignoring TabReformer, Holoclean outperformed the other techniques in the Flights and the Beers datasets with error $\%=2.5$. Comparably, Raha surpassed the other techniques, except for TabReformer, in the Adult and Beers datasets with an error rate $=10 \%$. Generally, the sensitivity analysis shows that the proposed method attains better performance than the other techniques with different error rates. Moreover, with lower rates of errors, TabReformer can significantly outperform the machine learning models without requiring additional training data.

4.5.2 Error Type Ratios Impact. Finally, to estimate the effectiveness of the proposed method to detect different error types, we conduct another set of experiments focusing on specific types 


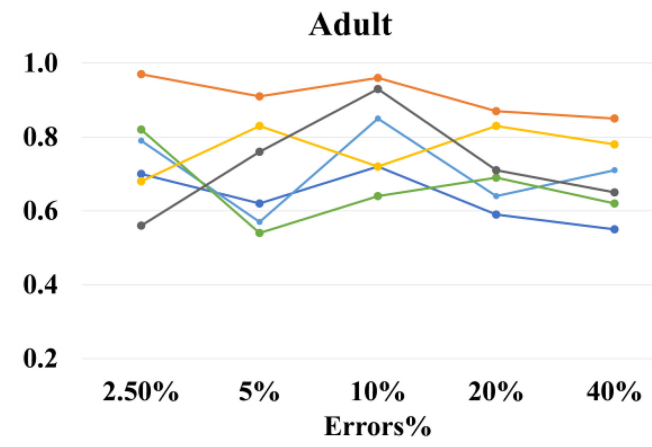

Flights

1.0

0.8

0.6

0.4

0.2

1.0

0.8

0.6

0.4

0.2
1.0

0.8

0.6

0.4

0.2

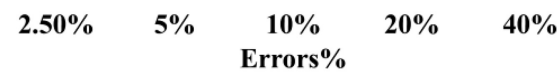

Movies

1.0

0.8

0.6

0.4

0.2

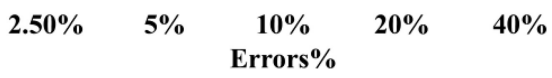

Beers

1.0

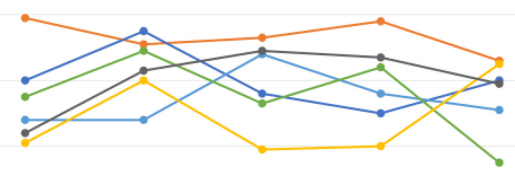

0.4

0.2

$2.50 \% \quad 5 \% \quad \begin{array}{cccc}10 \% & 20 \% & 40 \%\end{array}$

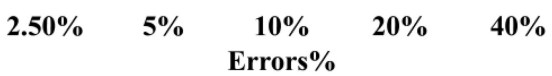

Errors\%

- TabReformer $*$ HoloClean -ED2 $*$ NADEEF $*$ ActiveClean $*$ Raha

Fig. 6. Performance of error detection methods with various error rates.

of errors with different ratios. As mentioned earlier, we consider five types of errors in the experiments: typographical errors, value swapped across columns, values violating data constraints, formatting errors, and missing values. Therefore, we repeated the experiments in Section 4.1 five times. On each occasion, we inject one type of error in each dataset with different rates $(5 \%, 15 \%$, and $30 \%$ ). To ensure data replicability, we repeated each test five times and reported the average (arithmetic mean) of the achieved F1 measure. The datasets used in the experiments are publicly released for future research ${ }^{1}$ and to allow for replicability.

The results obtained from the experiments are illustrated in Figure 7. For each error type, the figure shows the values of the F1 measure achieved by TabReformer, along with the competing tools. First, as for the errors originating from formatting issues, most of these injected errors

\footnotetext{
${ }^{1}$ https://github.com/MonaNashaat/TabReformer.
} 

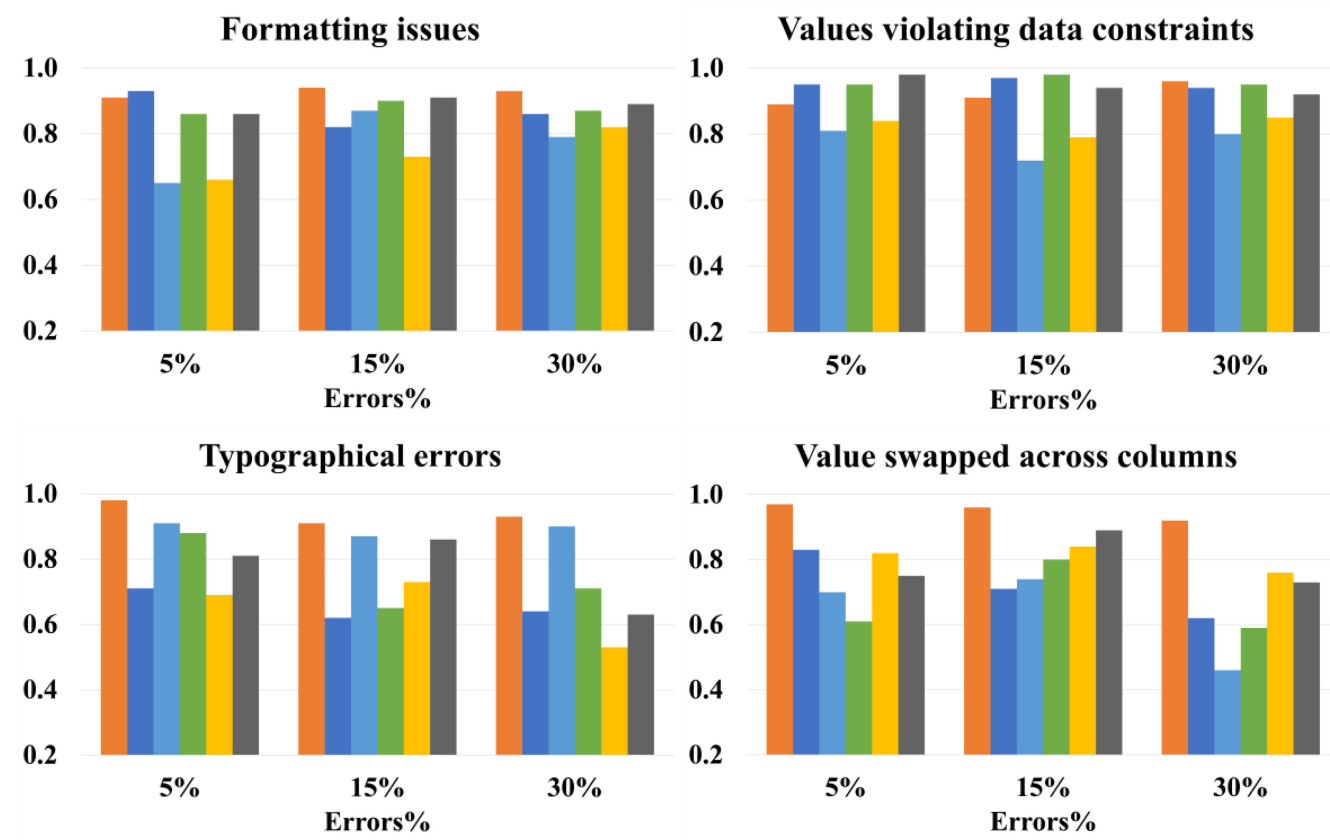

Value swapped across columns
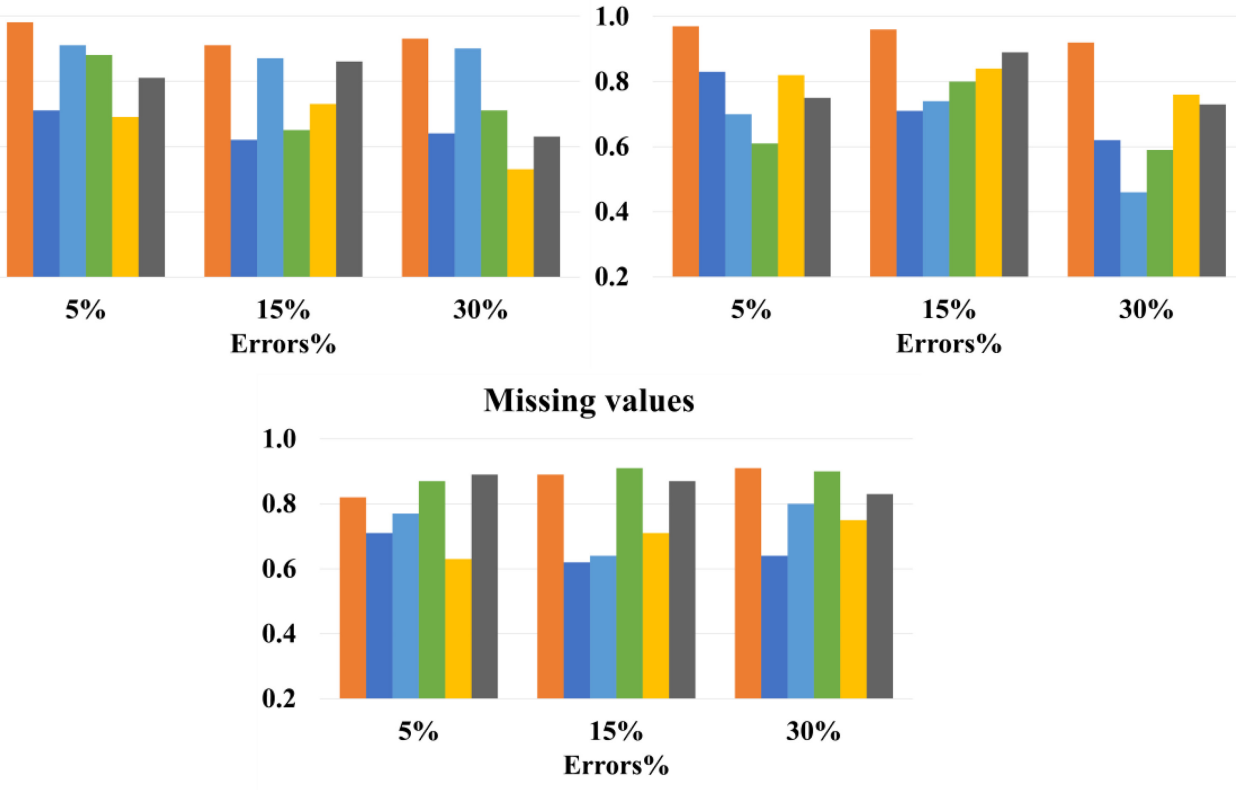

$\because$ TabReformer $\square$ HoloClean ED2 $\backsim$ NADEEF $\square$ ActiveClean $\square$ Raha

Fig. 7. Performance of error detection methods with various error types.

are related to date, time, year, and URL formats. The results show that TabReformer sustains an acceptable performance with various levels of error rates. Also, since integrity constraints can help detect most of these errors (e,g., date and time formats), rules-based systems such as NADEEF can achieve competitive performance. Similarly, the constraint violation detection algorithms embedded in HoloClean and Raha can detect most of these errors. Generally, the results show that even though TabReformer does not require the user to input any denial constraints, it can outperform most of the competing tools and maintain its superiority with higher error rates.

Second, the results show the same pattern with data constraints violation errors. Rules-based systems and learning models that rely on combining signals from different error detection algorithms can successfully detect the violating cells. For example, HoloClean achieved the same F1 measure as TabReformer and can surpass the competing tools with datasets with $30 \%$ violating cells. 
Alternatively, when dealing with typos and value swapping errors, NADEEF fails to detect most of these errors since they cannot be detected by integrity rules. Likewise, HoloClean achieves the worst performance among the other tools when dealing with a high rate of typographical errors (25\%). On the other hand, TabReformer can outperform most competing tools when dealing with value swapping errors. As the model is trained with the MDM objective to learn the data representation of each cell value while considering the values of all cells appearing before and after it, the model can detect the swapped values that do not fit into the tuple context. Finally, TabReformer achieves a competitive performance in detecting missing values when compared to rule-based systems. For example, it outperforms ED2 and Raha with $13.75 \%$ and $21.33 \%$, respectively, when dealing with a high rate of missing values. However, with fewer missing values (error ratio $=5 \%$ ), HoloClean achieves better F1 values than the other tools, with an average improvement of $19.95 \%$.

All in all, the results show that TabReformer can detect a wide range of errors with different ratios. The method can employ the learned representation to detect cells violating data constraints and formatting errors without defining denial constraints or data quality rules. For challenging error types, such as typographical errors and errors originating from swapped values, the method outperforms state-of-the-art techniques, with an improvement of $23.41 \%$ on average.

\subsection{Discussions}

TabReformer is a pretraining framework for erroneous data detection in tabular datasets. Although it uses transformer networks that are related to other NLP and language modeling methods [2325] including BERT [23], the proposed framework is fundamentally different from these methods. First, BERT applies two pretraining tasks (MLM and next sentence prediction) to understand the unstructured text. TabReformer, on the other hand, targets learning bidirectional encoder representation for tabular data by extracting intercolumn relationships between attributes regardless of their order. Therefore, applying self-attention heads allows TabReformer to detect the essential attributes for a given query vector (q) in a tuple. Second, BERT was trained on Wikipedia and Book Corpus, comprising more than 10,000 different books. The considerable computational resources required to train BERT allow researchers to use BERT's released models and subsequently fine-tune them on specific tasks. Most of these tasks utilize BERT's ability to extract highquality language features, such as language translation [26] and relation classification [27]. Finally, TabReformer allows the encoder to examine other cells in the input tuple as it generates the representation of a specific cell. As a result, the model can easily detect errors in cells that violate integrity constraints and functional dependencies.

Also, one of the critical reasons for the promising performance of TabReformer is the pretraining phase, which allows the model to understand the data representations of the structured data. Therefore, studying the effect of incorporating our trained model into the workflow of different baselines can be a viable research question and sensible directions for future work. For example, since error detection is considered a first step in the workflow of HoloClean, we can integrate the pretrained model of TabReformer into Holoclean as one of its error detection methods. Then again, Raha proposes an algorithm to fuse the output of different error detection strategies. Hence, with some engineering efforts, TabReformer can be utilized within Raha's workflow as one strategy of the error detection strategies for tabular data.

\section{RELATED WORK}

Given the fact that data-oriented approaches such as analytic systems are becoming critical for innovation in the enterprise, prior research explores a diverse set of techniques to detect and repair data quality issues. Also, since the focus of this research expands to different deep learning 
techniques, including data augmentation and unsupervised training, we survey existing effort regarding these areas with tabular data.

Learning models for erroneous data detection. Many studies [10, 63, 64] utilize machine learning techniques for error detection and data repairing tasks. As for error detection, recent research [63, 64] has applied machine learning for outlier detection. For example, Adeli et al. [64] propose a semi-supervised classification model to discriminate sample outliers and data noise. The model [64] estimates the noisy model using linear discriminant analysis and a labeled training dataset. However, most of these efforts [10, 63, 64] focus only on specific error categories. Furthermore, most of these approaches are applicable to specific domains only, such as computer vision [63] and medical imaging [64].

Other research $[65,66]$ has applied different learning paradigms to extract functional dependencies [66] and discover denial constraints [65]. One example is the approach presented in [65] to produce a probabilistic model that can induce functional dependencies. The model applies structural expectation-maximization to discover data rules and detect violating data. Unlike TabReformer, these techniques $[65,66]$ try to assist data analysts by formulating the integrity constraints from the data. Nevertheless, they do not provide fully automated error detection or data repairing systems.

Another example is SCODED [67], which leverages approximate statistical constraints from the data. After detecting the violating data, the model applies data partitioning to identify the minimum number of records that, if removed, could resolve detected violations. However, unlike the proposed model, the system relies on many assumptions regarding the underlying data characteristics. For example, SCODED [67] considers only multi-column dependencies and, hence, can be limited to single-column errors.

Data augmentation. To overcome the scarcity of training data, prior research [37, 68-70] has applied data augmentation to prevent overfitting. Popular techniques [68] apply affine transformations such as translation, relation, cropping, etc., to infer synthetic labeled images from actual images. Furthermore, recent research [37, 69] has utilized generative models to interpolate augmented examples from training data. For example, the authors in [69] propose a technique in which a generative adversarial network is first trained using the original data. Then, the learning is transferred to generate additional images. Another study [70] integrates data augmentation with semi-supervised learning and learns a model to treat augmented data as noise. As a result, the final model [70] is trained to become robust to input noise. However, these approaches are only applied to specific domains only, such as computer vision [69, 70], unstructured data [70], and audio separation [37]. Furthermore, none of these techniques have applied data augmentation to structured databases.

Self-supervised learning. Unsupervised representation learning aims to discover data characteristics without labeled examples. For example, to learn image representation, self-supervised learning can help the model to infer different relations between images during training. An existing approach [71] applies autoencoder networks to encode input features into representations that preserve the structure of the original images. The model is trained to predict the original label from the encoded features.

The same idea is employed in different domains, such as language modeling [23, 43] and audio classification [72]. However, unlike TabReformer, none of these approaches have considered tabular data or relational databases. Based on our literature survey, only one recent technique [73] has explored applying self-supervised learning to tabular data. Nevertheless, the technique mainly aims to learn decision-tree-like mappings of the data. As far as we know, no previous research has investigated the capability of attentive neural networks to classify erroneous data. 


\section{CONCLUSIONS}

The article presents TabReformer, a learning framework for detecting erroneous values in tabular data. The framework trains a bidirectional model to learn the data representation. To effectively achieve a deeper understanding of the data context, the model implements unsupervised representation learning using the Masked Data Model objective with GELU activation functions. To fine-tune the model, TabReformer introduces a phase of data augmentation to generate synthetic labeled examples while optimizing manual labeling effort. In the data augmentation process, both the transformation functions and the augmentation strategy are inferred from the underlying data with no need for any user-defined parameters. We evaluate the proposed framework by comparing its performance with state-of-the-art techniques for error detection and data repair. The empirical results show that TabReformer can significantly enhance the classification performance of erroneous values by up to $61.41 \%$ (F1 measure) while reducing the manual labeling budget by $31.77 \%$ on average. The experimental evaluation also depicts that the implemented data augmentation strategy outperforms other sampling techniques, such as active learning strategies and traditional resampling approaches. Overall, the results empirically prove that TabReformer can detect a diverse set of errors, tolerate high noise ratios, and surpass existing error detection techniques.

\section{REFERENCES}

[1] R. Lu, X. Jin, S. Zhang, M. Qiu, and X. Wu. 2019. A study on big knowledge and its engineering issues. IEEE Transactions on Knowledge and Data Engineering 31, 9 (2019), 1630-1644, DOI:10.1109/TKDE.2018.2866863.

[2] X. Chu, I. F. Ilyas, and P. Papotti. 2013. Holistic data cleaning: Putting violations into context. In 2013 IEEE 29th International Conference on Data Engineering (ICDE), 458-469, DOI:10.1109/ICDE.2013.6544847.

[3] T. Rekatsinas, X. Chu, I. F. Ilyas, and C. Ré. 2017. HoloClean: Holistic data repairs with probabilistic inference. Proceedings of the VLDB Endowment 10, 1 (2017).

[4] A. Reddy et al. 2017. Using gaussian mixture models to detect outliers in seasonal univariate network traffic. In 2017 IEEE Security and Privacy Workshops (SPW), 229-234, D0I:10.1109/SPW.2017.9.

[5] C. Pit-Claudel, Z. Mariet, R. Harding, and S. Madden. 2016. Outlier detection in heterogeneous datasets using automatic tuple expansion. 2016.

[6] F. Riahi and O. Schulte. 2020. Model-based exception mining for object-relational data. Data Mining and Knowledge Discovery 34, 3 (2020), 681-722, DOI:10.1007/s10618-020-00677-w.

[7] Y. Liu et al. 2019. Generative adversarial active learning for unsupervised outlier detection. IEEE Transactions on Knowledge and Data Engineering 2019, DOI:10.1109/TKDE.2019.2905606.

[8] S. Schelter, D. Lange, P. Schmidt, M. Celikel, F. Biessmann, and A. Grafberger. 2018. Automating large-scale data quality verification. Proceedings of the VLDB Endowment 11, 12 (2018), 1781-1794.

[9] M. Dallachiesa et al. 2013. NADEEF: A commodity data cleaning system. In Proceedings of the 2013 ACM SIGMOD International Conference on Management of Data, New York, NY, 2013, 541-552, DOI:10.1145/2463676. 2465327.

[10] L. Koumarelas, T. Papenbrock, and F. Naumann. 2020. MDedup: Duplicate detection with matching dependencies. Proceedings of the VLDB Endowment 13, 5 (2020), 712-725, DOI:10.14778/3377369.3377379.

[11] E. H. M. Pena, E. C. de Almeida, and F. Naumann. 2019. Discovery of approximate (and exact) denial constraints. Proceedings of the VLDB Endowment 13, 3 (2019), 266-278, DOI:10.14778/3368289.3368293.

[12] X. Chu, I. F. Ilyas, S. Krishnan, and J. Wang. 2016. Data cleaning: Overview and emerging challenges. In Proceedings of the 2016 International Conference on Management of Data, New York, NY, 2016, 2201-2206, DOI:10.1145/2882903. 2912574.

[13] Z. Abedjan et al. 2016. Detecting data errors: Where are we and what needs to be done? Proceedings of the VLDB Endowment 9, 12 (2016), 993-1004, 2016, DOI:10.14778/2994509.2994518.

[14] J. Yang, S. Rahardja, and P. Fränti. 2019. Outlier Detection: How to Threshold Outlier Scores?, In Proceedings of the International Conference on Artificial Intelligence, Information Processing and Cloud Computing, New York, NY, 2019, DOI:10.1145/3371425.3371427.

[15] A. Heidari, J. McGrath, I. F. Ilyas, and T. Rekatsinas. 2019. HoloDetect: Few-shot learning for error detection. In Proceedings of the 2019 International Conference on Management of Data, Netherlands, 2019, 829-846, DOI:10.1145/ 3299869.3319888 . 
[16] F. Neutatz, M. Mahdavi, and Z. Abedjan. 2019. ED2: A case for active learning in error detection. In Proceedings of the 28th ACM International Conference on Information and Knowledge Management, New York, NY, 2019, 2249-2252, DOI:10.1145/3357384.3358129.

[17] S. Krishnan, J. Wang, E. Wu, M. J. Franklin, and K. Goldberg. 2016. ActiveClean: Interactive data cleaning for statistical modeling. Proceedings of the VLDB Endowment 9, 12 (2016), 948-959, DOI:10.14778/2994509.2994514.

[18] S. Krishnan, M. J. Franklin, K. Goldberg, and E. Wu. 2017. BoostClean: Automated error detection and repair for machine learning. arXiv:1711.01299 [cs], 2017.

[19] A. Vaswani et al. 2017. Attention is all you need. In Advances in Neural Information Processing Systems, Curran Associates, Inc., 2017, 5998-6008.

[20] J. K. Chorowski, D. Bahdanau, D. Serdyuk, K. Cho, and Y. Bengio. 2015. Attention-based models for speech recognition. In Advances in Neural Information Processing Systems, Curran Associates, Inc., 2015, 577-585.

[21] J. Krantz and J. Kalita. 2018. Abstractive summarization using attentive neural techniques. arXiv:1810.08838 [cs], Oct. 2018.

[22] A. Sternberg, J. Soares, D. Carvalho, and E. Ogasawara. 2017. A review on flight delay prediction. arXiv:1703.06118 [cs], 2017.

[23] J. Devlin, M.-W. Chang, K. Lee, and K. Toutanova. 2019. BERT: Pre-training of deep bidirectional transformers for language understanding. arXiv:1810.04805 [cs], 2019.

[24] A. Adhikari, A. Ram, R. Tang, and J. Lin. 2019. DocBERT: BERT for document classification. arXiv:1904.08398 [cs], Aug. 2019 [Online]. Available: http://arxiv.org/abs/1904.08398.

[25] Z. Lan, M. Chen, S. Goodman, K. Gimpel, P. Sharma, and R. Soricut. 2020. ALBERT: A lite BERT for self-supervised learning of language representations. arXiv:1909.11942 [cs], 2020 [Online]. Available: http://arxiv.org/abs/1909.11942.

[26] K. Ahmed, N. S. Keskar, and R. Socher. 2017. Weighted transformer network for machine translation. arXiv:1711.02132 [cs], Nov. 2017 [Online]. Available: http://arxiv.org/abs/1711.02132.

[27] L. Fu, Z. Yin, Y. Liu, and J. Zhang. 2018. Convolution neural network with active learning for information extraction of enterprise announcements. In Natural Language Processing and Chinese Computing, Cham 2018, 330-339.

[28] M. R. A. Rashid, G. Rizzo, M. Torchiano, N. Mihindukulasooriya, O. Corcho, and R. García-Castro. 2019. Completeness and consistency analysis for evolving knowledge bases. fournal of Web Semantics 54, 48-71.

[29] M. Farid, A. Roatis, I. F. Ilyas, H.-F. Hoffmann, and X. Chu. 2016. CLAMS: Bringing quality to data lakes. In Proceedings of the 2016 International Conference on Management of Data, San Francisco, CA, 2016, 2089-2092, DOI:10.1145/2882903. 2899391.

[30] H. Saxena, L. Golab, and I. F. Ilyas. 2019. Distributed discovery of functional dependencies. In 2019 IEEE 35th International Conference on Data Engineering, Macao, 2019, 1590-1593, DOI:10.1109/ICDE.2019.00149.

[31] E. K. Rezig, M. Ouzzani, W. G. Aref, A. K. Elmagarmid, and A. R. Mahmood. 2017. Pattern-driven data cleaning. ArXiv:1712.09437 [cs], 2017.

[32] A. Qahtan, N. Tang, M. Ouzzani, Y. Cao, and M. Stonebraker. 2020. Pattern functional dependencies for data cleaning. Proceedings of the VLDB Endowment 13, 5 (2020), 684-697.

[33] Z. Abedjan, L. Golab, and F. Naumann. 2015. Profiling relational data: A survey. The VLDB fournal 24, 4 (2015), 557581, DOI:10.1007/s00778-015-0389-y.

[34] E. D. Cubuk, B. Zoph, J. Shlens, and Q. V. Le. 2019. RandAugment: Practical automated data augmentation with a reduced search space. arXiv:1909.13719 [cs], Nov. 2019.

[35] E. D. Cubuk, B. Zoph, D. Mane, V. Vasudevan, and Q. V. Le. 2019. AutoAugment: Learning augmentation strategies from data. Long Beach, CA, 2019, 113-123.

[36] B. Zoph and Q. V. Le. 2017. Neural architecture search with reinforcement learning. arXiv:1611.01578 [cs], 2017 [Online]. Available: http://arxiv.org/abs/1611.01578.

[37] D. Stoller, S. Ewert, and S. Dixon. 2018. Adversarial semi-supervised audio source separation applied to singing voice extraction. In 2018 IEEE International Conference on Acoustics, Speech and Signal Processing (ICASSP), Calgary, Alberta, Canada (2018), 2391-2395, DOI:10.1109/ICASSP.2018.8461722.

[38] S. Lim, I. Kim, T. Kim, C. Kim, and S. Kim. 2019. Fast autoaugment. In Advances in Neural Information Processing Systems, Curran Associates, Inc., 2019, 6665-6675.

[39] Y. Li, G. Hu, Y. Wang, T. Hospedales, N. M. Robertson, and Y. Yang. 2003. DADA: Differentiable automatic data augmentation. arXiv:2003.03780 [cs], 2020, [Online]. Available: http://arxiv.org/abs/2003.03780.

[40] D. Hendrycks and K. Gimpel. 2018. Gaussian error linear units (GELUs). arXiv:1606.08415 [cs], 2018 [Online]. Available: http://arxiv.org/abs/1606.08415.

[41] J. Torres, C. Vaca, L. Terán, and C. L. Abad. 2020. Seq2Seq models for recommending short text conversations. Expert Systems with Applications 150, 2020, DOI:10.1016/j.eswa.2020.113270.

[42] Z. Dai, Z. Yang, Y. Yang, J. Carbonell, Q. Le, and R. Salakhutdinov. 2019. Transformer-XL: Attentive language models beyond a fixed-length context. In Proceedings of the 57th Annual Meeting of the Association for Computational Linguistics, Florence, Italy (2019), 2978-2988, DOI:10.18653/v1/P19-1285. 
[43] Y. Liu et al. 2019. RoBERTa: A robustly optimized BERT pretraining approach. arXiv:1907.11692 [cs], Jul. 2019 [Online]. Available: http://arxiv.org/abs/1907.11692.

[44] Z. Lan, M. Chen, S. Goodman, K. Gimpel, P. Sharma, and R. Soricut. 2020. ALBERT: A Lite BERT for self-supervised learning of language representations. arXiv:1909.11942 [cs], 2020 [Online]. Available: http://arxiv.org/abs/1909.11942.

[45] J. T. Hancock and T. M. Khoshgoftaar. 2020. Survey on categorical data for neural networks. Fournal of Big Data 7, 1 (2020), DOI:10.1186/s40537-020-00305-w.

[46] H. Nam and H.-E. Kim. 2018. Batch-instance normalization for adaptively style-invariant neural networks. In Advances in Neural Information Processing Systems, Curran Associates, Inc. (2018), 2558-2567.

[47] D. Ulyanov, A. Vedaldi, and V. Lempitsky. 2017. Instance normalization: The missing ingredient for fast stylization. arXiv:1607.08022 [cs], 2017 [Online]. Available: http://arxiv.org/abs/1607.08022.

[48] W. L. Taylor. 1953. 'Cloze Procedure': A new tool for measuring readability. fournalism Quarterly 30, 4 (1953), 415433, DOI:10.1177/107769905303000401.

[49] B. Alipour, L. Tonetto, R. Ketabi, A. Yi Ding, J. Ott, and A. Helmy. 2019. Where are you going next? A practical multi-dimensional look at mobility prediction. In Proceedings of the 22nd International ACM Conference on Modeling, Analysis and Simulation of Wireless and Mobile Systems, New York, NY, 2019, 5-12, DOI:10.1145/3345768.3355923.

[50] P. Dun, L. Zhu, and D. Zhao. 2019. Extending answer prediction for deep bi-directional transformers. In 32nd Conference on Neural Information Processing Systems (NIPS'19).

[51] H. Tan and M. Bansal. 2019. LXMERT: Learning cross-modality encoder representations from transformers. arXiv preprint arXiv:1908.07490, 2019.

[52] F. Neutatz, M. Mahdavi, and Z. Abedjan. 2019. ED2: Two-stage active learning for error detection - technical report. arXiv:1908.06309 [cs, stat], Aug. 2019, Accessed: Apr. 17, 2020 [Online]. Available: http://arxiv.org/abs/1908.06309.

[53] N. S. Tawfik and M. R. Spruit. 2020. Evaluating sentence representations for biomedical text: Methods and experimental results. Journal of Biomedical Informatics 104, 103396 (2020), DOI:10.1016/j.jbi.2020.103396.

[54] M. Mahdavi et al. 2019. Raha: A configuration-free error detection system. In Proceedings of the 2019 International Conference on Management of Data, New York, NY (2019), 865-882, DOI:10.1145/3299869.3324956.

[55] D. Crane. "The Cost of Bad Data," Integrate, Inc, 201AD [Online]. Available: https://demand.integrate.com/rs/951JPP-414/images/Integrate_TheCostofBadLeads_Whitepaper.pdf.

[56] D. W. Cearley. 2020. Top 10 strategic technology trends for 2020, Gartner, 2020 [Online]. Available: https://www. gartner.com/en/publications/top-tech-trends-2020.

[57] D. Dua and C. Graff. 2017. UCI Machine Learning Repository. University of California, Irvine, School of Information and Computer Sciences, 2017.

[58] P. Wang and Y. He. 2019. Uni-detect: A unified approach to automated error detection in tables. In Proceedings of the 2019 International Conference on Management of Data, New York, NY, 2019, 811-828, DOI:10.1145/3299869. 3319855.

[59] Y. Fu, X. Zhu, and B. Li. 2013. A survey on instance selection for active learning. Knowledge and Information Systems 35, 2 (2013), 249-283, DOI:10.1007/s10115-012-0507-8.

[60] D. P. Kingma and J. Ba. 2017. Adam: A method for stochastic optimization. arXiv:1412.6980 [cs], Jan. 2017 [Online]. Available: http://arxiv.org/abs/1412.6980.

[61] P. Zhang, X. Xu, and D. Xiong. 2018. Active learning for neural machine translation. In 2018 International Conference on Asian Language Processing (IALP), 2018, 153-158.

[62] A. Estabrooks, T. Jo, and N. Japkowicz. 2004. A multiple resampling method for learning from imbalanced data sets. Computational Intelligence 20, 1 (2004), 18-36, DOI:10.1111/j.0824-7935.2004.t01-1-00228.x.

[63] S. Akcay, A. Atapour-Abarghouei, and T. P. Breckon. 2019. GANomaly: Semi-supervised anomaly detection via adversarial training. In Computer Vision - ACCV 2018, 2019, 622-637.

[64] E. Adeli et al. 2019. Semi-supervised discriminative classification robust to sample-outliers and feature-noises. IEEE Transactions on Pattern Analysis and Machine Intelligence 41, 2 (2019), 515-522.

[65] S. Eduardo and C. Sutton. 2016. Data cleaning using probabilistic models of integrity constraints. Neural Information Processing Systems.

[66] G. Zhu, Q. Wang, Q. Tang, R. Gu, C. Yuan, and Y. Huang. 2019. Efficient and scalable functional dependency discovery on distributed data-parallel platforms. IEEE Transactions on Parallel and Distributed Systems 30, 12 (2019), 2663-2676.

[67] J. N. Yan, O. Schulte, M. Zhang, J. Wang, and R. Cheng. 2020. SCODED: Statistical constraint oriented data error detection. Presented at the SIGMOD'20, Portland, OR, 2020.

[68] K. Chaitanya, N. Karani, C. F. Baumgartner, A. Becker, O. Donati, and E. Konukoglu. 2019. Semi-supervised and task-driven data augmentation. In Information Processing in Medical Imaging, 2019, 29-41.

[69] S. Liu, J. Zhang, Y. Chen, Y. Liu, Z. Qin, and T. Wan. 2019. Pixel level data augmentation for semantic image segmentation using generative adversarial networks. In 2019 IEEE International Conference on Acoustics, Speech and Signal Processing (ICASSP) (2019), 1902-1906, DOI:10.1109/ICASSP.2019.8683590. 
[70] Q. Xie, Z. Dai, E. Hovy, M.-T. Luong, and Q. V. Le. 2020. Unsupervised Data Augmentation for Consistency Training. arXiv 1904.12848v6 [csLG], 2020 [Online]. Available: https://arxiv.org/abs/1904.12848.

[71] L. Zhang, G.-J. Qi, L. Wang, and J. Luo. 2019. AET vs. AED: Unsupervised representation learning by auto-encoding transformations rather than data. In Proceedings of the IEEE Conference on Computer Vision and Pattern Recognition, USA, 2019, 2547-2555.

[72] M. Freitag, S. Amiriparian, S. Pugachevskiy, N. Cummins, and B. Schuller. 2017. AuDeep: Unsupervised learning of representations from audio with deep recurrent neural networks. The fournal of Machine Learning Research 18, 1 (2017), 6340-6344.

[73] S. O. Arik and T. Pfister. 2020. TabNet: Attentive interpretable tabular learning. arXiv:1908.07442 [cs, stat], Feb. 2020 [Online]. Available: http://arxiv.org/abs/1908.07442.

Received July 2020; revised January 2021; accepted January 2021 